\title{
LARGE DEVIATION PRINCIPLE OF FREIDLIN-WENTZELL TYPE FOR PINNED DIFFUSION PROCESSES
}

\author{
YUZURU INAHAMA
}

\begin{abstract}
Since T. Lyons invented rough path theory, one of its most successful applications is a new proof of Freidlin-Wentzell's large deviation principle for diffusion processes. In this paper we extend this method to the case of pinned diffusion processes under a mild ellipticity assumption. Besides rough path theory, our main tool is quasi-sure analysis, which is a kind of potential theory in Malliavin calculus.
\end{abstract}

\section{INTRODUCTION}

For the canonical realization of $d$-dimensional Brownian motion $\left(w_{t}\right)_{0 \leq t \leq 1}$ and the vector fields $V_{i}: \mathbf{R}^{n} \rightarrow \mathbf{R}^{n}(1 \leq i \leq d)$ with sufficient regularity, let us consider the following Stratonovich-type stochastic differential equation (SDE):

$$
d y_{t}=\sum_{i=1}^{d} V_{i}\left(y_{t}\right) \circ d w_{t}^{i} \quad \text { with } \quad y_{0}=a \in \mathbf{R}^{n} .
$$

For the sake of simplicity, no drift term is added here, but modification is easy. The correspondence $w \mapsto y$ is called the Itô map and denoted by $y=\Phi(w)$. It is well known that the Itô map is not continuous as a map from the Wiener space. Moreover, it is not continuous with respect to any Banach norm on the Wiener space which preserves the structure of the Wiener space (see Sugita [27]).

Now, introduce a small positive parameter $\varepsilon \in(0,1]$ and consider

$$
d y_{t}^{\varepsilon}=\sum_{i=1}^{d} V_{i}\left(y_{t}^{\varepsilon}\right) \circ \varepsilon d w_{t}^{i} \quad \text { with } \quad y_{0}^{\varepsilon}=a \in \mathbf{R}^{n} .
$$

Formally, $y^{\varepsilon}=\Phi(\varepsilon w)$. The process $\left(y_{t}^{\varepsilon}\right)_{0 \leq t \leq 1}$ takes its values in $\mathbf{R}^{n}$ and its law is a diffusion measure associated with the starting point $a$ and the generator $\mathcal{L}^{\varepsilon}=$ $\left(\varepsilon^{2} / 2\right) \sum_{i=1}^{d} V_{i}^{2}$.

A classic result of Freidlin and Wentzell states the laws of $\left(y_{t}^{\varepsilon}\right)_{0 \leq t \leq 1}$ satisfy a large deviation principle as $\varepsilon \searrow 0$. The proof was not so easy. (See Friedman [6] or Dembo-Zeitouni [4, for instance.) If $\Phi$ were continuous, we could use the contraction principle and the proof would be immediate from Schilder's large deviation principle for the laws of $\left(\varepsilon w_{t}\right)_{0 \leq t \leq 1}$. However, it cannot be made continuous in the framework of the usual stochastic analysis.

Received by the editors April 1, 2013 and, in revised form, September 6, 2013.

2010 Mathematics Subject Classification. Primary 60F10, 60H07, 60H99, 60J60.

Key words and phrases. Large deviation principle, pinned diffusion process, stochastic differential equation, rough path theory, quasi-sure analysis. 
Ten years ago, Ledoux, Qian, and Zhang [18 gave a new proof by means of rough path theory, which was invented by T. Lyons [19. See also [10, 20], for example. Roughly speaking, a rough path is a couple of a path itself and its iterated integrals. Lyons established a theory of line integrals along rough paths and ordinary differential equation (ODE) driven by rough paths. The Itô map in the rough path sense is deterministic and is sometimes called the Lyons-Itô map. The most important result in the rough path theory could be Lyons' continuity theorem (also known as the universal limit theorem), which states that the Lyons-Itô map is continuous in the rough path setting.

Brownian motion $\left(w_{t}\right)$ admits a natural lift to a random rough path $W$, which is called a Brownian rough path. If we put $W$ or $\varepsilon W$ into the Lyons-Itô map, then we obtain the solution of Stratonovich SDE $\left(y_{t}\right)$ or $\left(y_{t}^{\varepsilon}\right)$, respectively. They proved in [18 that the laws of $\varepsilon W$ satisfy a large deviation principle of Schilder type with respect to the topology of the rough path space. The large deviation principle of Freidlin-Wentzell type for the laws of $\left(y_{t}^{\varepsilon}\right)$ is immediate from this, since the contraction principle can be used in this framework. Since then many works on the large deviation principle on a rough path space have been published [5, 8, 9, 16, 23].

Then, a natural question arises: can one obtain a similar result for pinned diffusion processes with this method, too? More precisely, does the family of measures $\left\{\mathbb{Q}_{a, a^{\prime}}^{\varepsilon}\right\}_{\varepsilon>0}$ satisfy a large deviation principle as $\varepsilon \searrow 0$ ? Here, $\mathbb{Q}_{a, a^{\prime}}^{\varepsilon}$ is the pinned diffusion measure associated with $\mathcal{L}^{\varepsilon}$, which starts at $a$ at time $t=0$ and ends at $a^{\prime}$ at time $t=1$. Heuristically, $\mathbb{Q}_{a, a^{\prime}}^{\varepsilon}$ is the law of $y_{1}^{\varepsilon}$ under the conditional probability measure $\mathbb{P}\left(\cdot \mid y_{1}^{\varepsilon}=a^{\prime}\right)$, where $\mathbb{P}$ stands for the Wiener measure.

The aim of this paper is to answer this question affirmatively under a certain mild ellipticity assumption for the coefficient vector fields. Besides rough path theory, our main tool is quasi-sure analysis, which is a sub-field of Malliavin calculus. It deals with objects such as Watanabe distributions (i.e., generalized Wiener functionals) and capacities associated with Sobolev spaces. Recall that motivation for developing this theory was to analyse (the pullbacks of) pinned diffusion measures on the Wiener space.

The organization of this paper is as follows. In Section 2, we give a precise setting, introduce assumptions, and state our main results. The most important among them is Theorem 2.1(i), which is a large deviation principle on the geometric rough path space. We also discuss preceding results. In Section 3, we introduce Besovtype norms on the geometric rough path space and prove their basic properties in relation to Hölder norms. In rough path theory, Hölder norms and variation norms are most important, while Besov norms merely play auxiliary roles. However, in connection with Malliavin calculus, Besov norms on the rough path space become very important. In Section 4, we give a brief survey of Malliavin calculus and quasi-sure analysis for later use. We give basic facts on capacities and Watanabe distributions. Sugita's theorem is the most important among them. It states that a positive Watanabe distribution is actually a finite Borel measure on the abstract Wiener space.

In Section 5, following Higuchi 12 and Aida 1], we recall that Brownian motion $w$ admits a natural lift quasi-surely and this version of the Brownian rough path $W$ is $\infty$-quasi-continuous. In Section 6 , using this quasi-continuity and Sugita's theorem, we obtain a probability measure on the geometric rough path space such that its pushforward by the Lyons-Itô map induces the pinned diffusion measure in question. 
Section 7 is the the main part of our efforts. In this section we prove our main results, in particular, Theorem 2.1 (i). Several key facts used in these sections are as follows: (1) large deviation estimate for capacities on geometric rough path space, (2) integration by parts formula in the sense of Malliavin calculus for Watanabe distributions, (3) uniform non-degeneracy of Malliavin covariance matrix for solutions of the shifted SDE.

\section{SEtTing AND MAin RESUlt}

In this section we give a precise setting and state our main result. Let $\left(w_{t}\right)_{0 \leq t \leq 1}$ be the canonical realization of $d$-dimensional Brownian motion. We consider the following $\mathbf{R}^{n}$-valued Stratonovich-type SDE:

$$
d y_{t}^{\varepsilon}=\sum_{i=1}^{d} V_{i}\left(y_{t}^{\varepsilon}\right) \circ \varepsilon d w_{t}^{i}+V_{0}\left(\varepsilon, y_{t}^{\varepsilon}\right) d t \quad \text { with } \quad y_{0}^{\varepsilon}=a \in \mathbf{R}^{n} .
$$

Here, $\varepsilon \in[0,1]$ is a small parameter and $V_{i} \in C_{b}^{\infty}\left(\mathbf{R}^{n}, \mathbf{R}^{n}\right)$ for $1 \leq i \leq d$ and $V_{0} \in C_{b}^{\infty}\left([0,1] \times \mathbf{R}^{n}, \mathbf{R}^{n}\right)$. (A function is said to be of class $C_{b}^{\infty}$ if it is a bounded smooth function with bounded derivatives of all order.) For each $\varepsilon,\left(y_{t}^{\varepsilon}\right)$ is a diffusion process with its generator

$$
\mathcal{L}^{\varepsilon}=\frac{\varepsilon^{2}}{2} \sum_{i=1}^{d} V_{i}^{2}+V_{0}(\varepsilon, \cdot)
$$

We denote by $\mathbb{Q}_{a, a^{\prime}}^{\varepsilon}$ the pinned diffusion measure associated with $\mathcal{L}^{\varepsilon}$ for $\varepsilon>0$, starting point $a$, and terminal point $a^{\prime}$ if it exists.

Let $\mathcal{H}$ be Cameron-Martin space for $\left(w_{t}\right)$. For $h \in \mathcal{H}$, we denote by $\phi^{0}=\phi^{0}(h)$ a unique solution of the following ODE:

$$
d \phi_{t}^{0}=\sum_{i=1}^{d} V_{i}\left(\phi_{t}^{0}\right) d h_{t}^{i}+V_{0}\left(0, \phi_{t}^{0}\right) d t \quad \text { with } \quad \phi_{0}^{0}=a .
$$

We set $\mathcal{K}^{a, a^{\prime}}=\left\{h \in \mathcal{H} \mid \phi^{0}(h)_{1}=a^{\prime}\right\}$.

Let $a, a^{\prime}$ be given. To exclude pathological cases, we assume the following:

(H0) $\mathcal{K}^{a, a^{\prime}}$ is not empty.

For the vector fields, we assume ellipticity only at the starting point $a$ :

(H1) The set of vectors $\left\{V_{1}(a), \ldots, V_{d}(a)\right\}$ linearly spans $\mathbf{R}^{n}$.

Under this assumption, the heat kernel $p_{t}^{\varepsilon}\left(a, a^{\prime}\right)$ exists for all $a^{\prime} \in \mathbf{R}^{n}, t>0$ and $\varepsilon>0$. Note that $p_{t}^{\varepsilon}\left(a, a^{\prime}\right)$ is continuous (actually smooth) in $a^{\prime}$. We also assume the following:

(H2) $p_{1}^{\varepsilon}\left(a, a^{\prime}\right)>0$ for any $0<\varepsilon \leq 1$.

Without (H2) the normalization is impossible and $\mathbb{Q}_{a, a^{\prime}}^{\varepsilon}$ or similar probability measures on the path space cannot be defined. If the vector fields $\left\{V_{1}, \ldots, V_{d}\right\}$ satisfy everywhere ellipticity, then (H0)-(H2) are satisfied.

Before we state our main results, let us introduce some notation. We will give detailed explanations for terminologies from rough path theory and Malliavin calculus in later sections. Let $G \Omega_{\alpha, 4 m}^{B}\left(\mathbf{R}^{d}\right)$ be the geometric rough path space with $(\alpha, 4 m)$-Besov topology. We assume the following condition on the Besov parameters $(\alpha, 4 m)$ :

$$
m \in \mathbf{N}_{+}, \quad \frac{1}{3}<\alpha<\frac{1}{2}, \quad \alpha-\frac{1}{4 m}>\frac{1}{3}, \quad \text { and } \quad 4 m-8 m \alpha>2 .
$$


Note that $S_{2}(\mathcal{H}) \subset G \Omega_{\alpha, 4 m}^{B}\left(\mathbf{R}^{d}\right)$, where $S_{2}$ stands for the natural (rough path) lift. Under (H1), the positive Watanabe distribution $\delta_{a^{\prime}}\left(y_{1}^{\varepsilon}\right)=\delta_{a^{\prime}}\left(y^{\varepsilon}(1, a)\right)$ is well defined. By Sugita's theorem, it is actually a finite Borel measure $\theta_{a, a^{\prime}}^{\varepsilon}$ on the Wiener space. Its lift, denoted by $\mu_{a, a^{\prime}}^{\varepsilon}=\left(\varepsilon S_{2}\right)_{*} \theta_{a, a^{\prime}}^{\varepsilon}$, is a finite Borel measure on $G \Omega_{\alpha, 4 m}^{B}\left(\mathbf{R}^{d}\right)$. Under (H2), the normalization of these measures is denoted by $\hat{\theta}_{a, a^{\prime}}^{\varepsilon}$ and $\hat{\mu}_{a, a^{\prime}}^{\varepsilon}$, respectively.

Set a rate function $I: G \Omega_{\alpha, 4 m}^{B}\left(\mathbf{R}^{n}\right) \rightarrow[0, \infty]$ as follows:

$$
I(X)= \begin{cases}\|h\|_{\mathcal{H}}^{2} / 2 & \left(\text { if } X=S_{2}(h) \text { for some } h \in \mathcal{K}^{a, a^{\prime}}\right), \\ \infty & \text { (otherwise) }\end{cases}
$$

This rate function $I$ is good. We also set $\hat{I}(X)=I(X)-\min \left\{\|h\|_{\mathcal{H}}^{2} / 2 \mid h \in \mathcal{K}^{a, a^{\prime}}\right\}$.

The following theorem is our main result in this paper. The proof will be given in Section 7.

Theorem 2.1. We keep the same notation and assumptions as above. Let $m \in \mathbf{N}_{+}$ and $1 / 3<\alpha<1 / 2$ be as in (2.3). Then, the following (i)-(ii) hold:

(i) Assume (Ho) and (H1). Then, the family $\left\{\mu_{a, a^{\prime}}^{\varepsilon}\right\}_{\varepsilon>0}$ of finite measures satisfies a large deviation principle on $G \Omega_{\alpha, 4 m}^{B}\left(\mathbf{R}^{d}\right)$ as $\varepsilon \searrow 0$ with a good rate function $I$, that is, for any Borel set $A \subset G \Omega_{\alpha, 4 m}^{B}\left(\mathbf{R}^{d}\right)$, the following inequalities hold:

$$
-\inf _{X \in A^{\circ}} I(X) \leq \liminf _{\varepsilon \searrow 0} \varepsilon^{2} \log \mu_{a, a^{\prime}}^{\varepsilon}(A) \leq \limsup _{\varepsilon \searrow 0} \varepsilon^{2} \log \mu_{a, a^{\prime}}^{\varepsilon}(A) \leq-\inf _{X \in \bar{A}} I(X) .
$$

(ii) Assume (H0)-(H2). Then, the family $\left\{\hat{\mu}_{a, a^{\prime}}^{\varepsilon}\right\}_{\varepsilon>0}$ of probability measures satisfies a large deviation principle on $G \Omega_{\alpha, 4 m}^{B}\left(\mathbf{R}^{d}\right)$ as $\varepsilon \searrow 0$ with a good rate function $\hat{I}$.

Note that Theorem 2.1 above also holds with respect to $\alpha^{\prime}$-Hölder geometric rough path topology for any $\alpha^{\prime} \in(1 / 3,1 / 2)$, since we can find $\alpha, m$ with (2.3) such that $(\alpha, 4 m)$-Besov topology is stronger than $\alpha^{\prime}$-Hölder topology.

Now we turn to the pinned diffusion measures. Under (H1) and (H2) the probability measure $\hat{\mu}_{a, a^{\prime}}^{\varepsilon}$ exists. Let $\Phi_{\varepsilon}$ be the Lyons-Itô map associated with $\left\{V_{1}, \ldots, V_{d} ; V_{0}(\varepsilon, \cdot)\right\} . \tau_{\lambda}$ stands for the Young pairing map with $\lambda_{t}=t$. Then, by the contraction principle and a slight generalization of Lyons' continuity theorem, $\left\{\left(a+\Phi_{\varepsilon}^{1} \circ \tau_{\lambda}\right)_{*} \hat{\mu}_{a, a^{\prime}}^{\varepsilon}\right\}_{\varepsilon>0}$ satisfies a large deviation principle on

$C_{a, a^{\prime}}^{\alpha-H}\left([0,1], \mathbf{R}^{n}\right)=\left\{x \in C\left([0,1], \mathbf{R}^{n}\right) \mid x\right.$ is $\alpha$-Hölder continuous, $\left.x_{0}=a, x_{1}=a^{\prime}\right\}$

for any $1 / 3<\alpha<1 / 2$. In fact, $\left(a+\Phi_{\varepsilon}^{1} \circ \tau_{\lambda}\right)_{*} \hat{\mu}_{a, a^{\prime}}^{\varepsilon}=\left(\tilde{y}^{\varepsilon}\right)_{*} \hat{\theta}_{a, a^{\prime}}^{\varepsilon}$, where $\tilde{y}^{\varepsilon}$ is an $\infty$-quasi redefinition of $y^{\varepsilon}$.

On the other hand, it is not easy to see whether the pinned diffusion measure in an elementary sense exists under (H1) and (H2). But, for instance, if we assume everywhere hypoellipticity for $\left\{V_{1}, \ldots, V_{d} ; V_{0}(\varepsilon, \cdot)\right\}$ for any $\varepsilon \in(0,1]$, then $\mathbb{Q}_{a, a^{\prime}}^{\varepsilon}$ exists and coincides with $\left(a+\Phi_{\varepsilon}^{1} \circ \tau_{\lambda}\right)_{*} \hat{\mu}_{a, a^{\prime}}^{\varepsilon}$. In other words, $\left(a+\Phi_{\varepsilon}^{1} \circ \tau_{\lambda}\right)_{*} \hat{\mu}_{a, a^{\prime}}^{\varepsilon}$ is a generalization of the pinned diffusion measure $\mathbb{Q}_{a, a^{\prime}}^{\varepsilon}$. (See Section 6 for details.)

Thus, as a corollary of Theorem 2.1] we have obtained a Freidlin-Wentzell type large deviation principle for pinned diffusion measures. Below we will state it explicitly. 
Define $J: C_{a, a^{\prime}}^{\alpha-H}\left([0,1], \mathbf{R}^{n}\right) \rightarrow[0, \infty]$ by

$$
J(y)=\inf \left\{\frac{\|h\|_{\mathcal{H}}^{2}}{2} \mid h \in \mathcal{K}^{a, a^{\prime}} \text { with } y=\phi^{0}(h)\right\}-\min \left\{\frac{\|h\|_{\mathcal{H}}^{2}}{2} \mid h \in \mathcal{K}^{a, a^{\prime}}\right\}
$$

if $y=\phi^{0}(h)$ for some $h \in \mathcal{K}^{a, a^{\prime}}$ and define $J(y)=\infty$ if no such $h \in \mathcal{K}^{a, a^{\prime}}$ exists. $J$ is also good.

Theorem 2.2. Let $1 / 3<\alpha<1 / 2$. In addition to (H0)-(H2), assume everywhere hypoellipticity for $\left\{V_{1}, \ldots, V_{d} ; V_{0}(\varepsilon, \cdot)\right\}$ for any $\varepsilon \in(0,1]$. The family $\left\{\mathbb{Q}_{a, a^{\prime}}^{\varepsilon}\right\}_{\varepsilon>0}$ of pinned diffusion measures on $C_{a, a^{\prime}}^{\alpha-H}\left([0,1], \mathbf{R}^{d}\right)$ satisfies a large deviation principle as $\varepsilon \searrow 0$ with a good rate function $J$, that is, for any Borel subset $A \subset C_{a, a^{\prime}}^{\alpha-H}\left([0,1], \mathbf{R}^{n}\right)$,

$$
-\inf _{y \in A^{\circ}} J(y) \leq \liminf _{\varepsilon \searrow 0} \varepsilon^{2} \log \mathbb{Q}_{a, a^{\prime}}^{\varepsilon}(A) \leq \limsup _{\varepsilon \searrow 0} \varepsilon^{2} \log \mathbb{Q}_{a, a^{\prime}}^{\varepsilon}(A) \leq-\inf _{y \in \bar{A}} J(y) .
$$

Remark 2.3. Let $F$ be any continuous map from $G \Omega_{\alpha, 4 m}^{B}\left(\mathbf{R}^{d}\right)$ to a Hausdorff topological space. Then, by the contraction principle, the family $\left\{F_{*} \mu_{a, a^{\prime}}^{\varepsilon}\right\}_{\varepsilon>0}$ of image measures satisfies a large deviation principle as $\varepsilon \searrow 0$. Roughly speaking, if we take the Lyons-Itô map determined by the vector fields $V_{i}$ 's, then we get Theorem 2.2. But, we may take a different Lyons-Itô map as $F$. This is worth mentioning since Theorem 2.1 [28] is formulated in such a way.

The rest of the section is devoted to looking at preceding results. It is clear that Theorem 2.1 is new, because the number of papers on rough paths is not so large yet. On the other hand, whether Theorem 2.2 is new is less clear since pinned diffusions have been extensively studied. However, it seems to the author that there is no explicitly written proof of this kind of large deviation.

In 1994 Takanobu and Watanabe presented this kind of large deviation principle under a hypoellipticity assumption for coefficient vector fields (Theorem 2.1, 28]). This result seems very general and nice, but they gave no proof. Their tool was also quasi-sure analysis. At that time rough path theory did not exist. Presumably, they computed Besov norm of the solution of SDE, but details are unknown. (Extending our method to the hypoelliptic case is an interesting and important future task.)

Another preceding result is by Hsu 13 for a special case. He proved the case for (scaled) Brownian bridge on a complete Reimannian manifold $M$ (i.e., the case $\mathcal{L}^{\varepsilon}=\left(\varepsilon^{2} / 2\right) \triangle_{M}$, where $\triangle_{M}$ stands for Laplace-Beltrami operator on $\left.M\right)$. His proof is based on estimates and asymptotics for the heat kernel of $\triangle_{M} / 2$, and no SDE appears in his paper. In this sense, this nice result of Hsu does not so much "precede" ours and it may not be very suitable to call it "Freidlin-Wentzell type". (After this work was done, the author was informed of Bailleul's result [2] Roughly speaking, this is a "hypoelliptic version" of Hsu's result 13 . His method is basically analytic, but he uses a little bit of rough path theory, too.)

A somewhat similar, but different result was obtained by Gao and Ren [11. They proved that the Freidlin-Wentzell large deviations hold for capacities on the Wiener space. It may be possible to prove that their result implies large deviations for pinned diffusions. The outline is as follows. (i) Combining a result in [11] with the known fact that measures of finite energy integral are dominated by a capacity shows that a measure of finite energy integral satisfies a Freidlin-Wentzell type estimate. (ii) Because the pinned diffusion measure is a measure of finite energy integral, it satisfies a Freidlin-Wentzell type estimate. (iii) The general principle 
of Baldi-Sanz [3] then implies that the pinned diffusion measure satisfies a large deviation principle. However, it is unclear under what kind of assumptions on vector fields each step above holds true since there is no detailed explanation.

Let us compare our method with the one in [11,28. In [11,28] they calculated with quasi-sure analysis the solution of SDE, which is the output of the Itô map. Since we use rough path theory, we will compute, not the output, but the input of the (Lyons-)Itô map. Here, the input means $\left(w_{t}\right)$ itself and its iterated Stratonovich stochastic integrals. Hence, we believe that our proof via rough paths is probably simpler.

On the other hand, we do not use the following. (i) Malliavin calculus for Banach space-valued Wiener functionals as in [11,22] and (ii) Kusuoka-Stroock's large deviation transfer method for a family of measurable maps (Theorem 2.1, [11], originally in Section 2, [17]). Since both of them are hard to understand even for those who are familiar with Malliavin calculus or large deviations, being able to avoid them could be called an advantage.

\section{Geometric rough path space with Besov norm}

In this section we introduce the geometric rough path space with Besov norm and recall its relation to the one with Hölder norm. Chapter 7 and Appendix A.2 in Friz and Victoir [10] may be a nice summary on this issue. In rough path theory, we usually use Hölder and variation norms. In connection to Malliavin calculus, however, Besov norm plays an essential role.

Throughout this paper, the time interval is $[0,1]$ and we set $\triangle=\{(s, t) \mid 0 \leq$ $s \leq t \leq 1\}$. For any $Y \in C\left(\triangle, \mathbf{R}^{d}\right)$, we set

$$
\|Y\|_{\alpha-H}=\sup _{0 \leq s<t \leq 1} \frac{\left|Y_{s, t}\right|}{|t-s|^{\alpha}} \quad(0<\alpha \leq 1)
$$

$$
\|Y\|_{\alpha, m-B}=\left(\iint_{0 \leq s<t \leq 1} \frac{\left|Y_{s, t}\right|^{m}}{|t-s|^{1+m \alpha}} d s d t\right)^{1 / m} \quad(m \geq 1,0<\alpha \leq 1) .
$$

These are called $\alpha$-Hölder norm and $(\alpha, m)$-Besov norm, respectively. There are some variants of Besov-type norms, but we will use this one.

Set $C^{\alpha-H}\left([0,1], \mathbf{R}^{d}\right)=\left\{x \in C\left([0,1], \mathbf{R}^{d}\right) \mid\left\|X^{1}\right\|_{\alpha-H}<\infty\right\}$, where $X_{s, t}^{1}:=$ $x_{t}-x_{s}$. This is called the space of $\mathbf{R}^{d}$-valued $\alpha$-Hölder continuous paths and becomes a real Banach space with $\|x\|=\left|x_{0}\right|+\left\|X^{1}\right\|_{\alpha-H}$. Its closed subset of paths that start at $a \in \mathbf{R}^{d}$ is denoted by $C_{a}^{\alpha-H}\left([0,1], \mathbf{R}^{d}\right)$. In a similar way, if $\alpha-m^{-1}>0, C^{\alpha, m-B}\left([0,1], \mathbf{R}^{d}\right)$, etc. are defined. Then, $C^{\alpha, m-B}\left([0,1], \mathbf{R}^{d}\right)$ is continuously embedded in $C^{\left(\alpha-m^{-1}\right)-H}\left([0,1], \mathbf{R}^{d}\right)$. (See Appendix A.2, [10].) In this case, closed subsets such as $C_{a}^{\alpha, m-B}\left([0,1], \mathbf{R}^{d}\right)$ are well defined.

Let $T^{2}\left(\mathbf{R}^{d}\right)=\mathbf{R} \oplus \mathbf{R}^{d} \oplus\left(\mathbf{R}^{d}\right)^{\otimes 2}$ be the truncated tensor algebra of Step 2. The set of elements of the form $(1, \bullet, \star)$ forms a non-abelian group under the tensor multiplication $\otimes$. The unit element is $\mathbf{1}=(1,0,0)$. Set

$$
G^{2}\left(\mathbf{R}^{d}\right)=\left\{\left(1, a_{1}, a_{2}\right) \in T^{2}\left(\mathbf{R}^{d}\right) \mid a_{2}^{i, j}+a_{2}^{j, i}=a_{1}^{i} a_{1}^{j} \quad(1 \leq i, j \leq d)\right\} .
$$

It is easy to check that $G^{2}\left(\mathbf{R}^{d}\right)$ becomes a subgroup. It is called the free nilpotent group of Step 2. Note that the dilation on $T^{2}\left(\mathbf{R}^{d}\right)$ (i.e., $\left(1, a_{1}, a_{2}\right) \mapsto\left(1, \lambda a_{1}, \lambda^{2} a_{2}\right)$ for $\lambda \in \mathbf{R})$ is well defined on $G^{2}\left(\mathbf{R}^{d}\right)$, too. 
A continuous map $X=\left(1, X^{1}, X^{2}\right): \triangle \rightarrow T^{2}\left(\mathbf{R}^{d}\right)$ is called multiplicative if it satisfies that

$$
X_{s, t}^{1}=X_{s, u}^{1}+X_{u, t}^{1}, \quad X_{s, t}^{2}=X_{s, u}^{2}+X_{u, t}^{2}+X_{s, u}^{1} \otimes X_{u, t}^{1} \quad(s \leq u \leq t) .
$$

This relation is called Chen's identity and can also be written as $X_{s, t}=X_{s, u} \otimes X_{u, t}$. In particular, $X_{s, t}(s \leq t)$ is a "difference" of a group-valued path, since $X_{s, t}=$ $\left(X_{0, s}\right)^{-1} \otimes X_{0, t}$.

Let $1 / 3<\alpha<1 / 2$. The space of $\mathbf{R}^{d}$-valued $\alpha$-Hölder rough path is defined by

$$
\begin{aligned}
\Omega_{\alpha}^{H}\left(\mathbf{R}^{d}\right)=\{ & X=\left(1, X^{1}, X^{2}\right) \in C\left(\triangle, T^{2}\left(\mathbf{R}^{d}\right)\right) \mid \\
& \text { multiplicative and } \left.\left\|X^{1}\right\|_{\alpha-H}<\infty,\left\|X^{2}\right\|_{2 \alpha-H}<\infty\right\} .
\end{aligned}
$$

The topology of this space is naturally induced by the following distance: $d(X, Y)=$ $\left\|X^{1}-Y^{1}\right\|_{\alpha-H}+\left\|X^{2}-Y^{2}\right\|_{2 \alpha-H}$. In the same way, $(\alpha, m)$-Besov rough path is defined for $m \geq 2$ and $1 / 3<\alpha<1 / 2$ with $\alpha-m^{-1}>1 / 3$ as follows:

$$
\begin{aligned}
\Omega_{m, \alpha}^{B}\left(\mathbf{R}^{d}\right)=\{ & X=\left(1, X^{1}, X^{2}\right) \in C\left(\triangle, T^{2}\left(\mathbf{R}^{d}\right)\right) \mid \\
& \text { multiplicative and } \left.\left\|X^{1}\right\|_{\alpha, m-B}<\infty,\left\|X^{2}\right\|_{2 \alpha, m / 2-B}<\infty\right\} .
\end{aligned}
$$

The topology of this space is naturally induced by the following distance: $d(X, Y)=$ $\left\|X^{1}-Y^{1}\right\|_{\alpha, m-B}+\left\|X^{2}-Y^{2}\right\|_{2 \alpha, m / 2-B}$. In what follows, we will often write $X=\left(X^{1}, X^{2}\right)$ for simplicity, since the 0th component " 1 " is obvious.

A Lipschitz continuous path (i.e., 1-Hölder continuous path) $x \in C_{0}^{1-H}\left([0,1], \mathbf{R}^{d}\right)$ admits a natural lift to a rough path by setting

$$
X_{s, t}^{1}:=x_{t}-x_{s}, \quad X_{s, t}^{2}:=\int_{s}^{t}\left(x_{u}-x_{s}\right) \otimes d x_{u}, \quad(s, t) \in \triangle .
$$

It is easy to see that $X \in \Omega_{\alpha}^{H}\left(\mathbf{R}^{d}\right) \cap \Omega_{\alpha, m}^{B}\left(\mathbf{R}^{d}\right)$. We call a rough path $X$ obtained in this way a smooth rough path lying above $x$, or the lift of $x$. The lift map is denoted by $S_{2}$, i.e., $X=S_{2}(x)$.

An $\alpha$-Hölder weakly geometric rough path is $X \in \Omega_{\alpha}^{H}\left(\mathbf{R}^{d}\right)$ such that

$$
X_{s, t}^{2 ; i, j}+X_{s, t}^{2 ; j, i}=X_{s, t}^{1 ; i} X_{s, t}^{1 ; j} \quad(1 \leq i, j \leq d, \quad(s, t) \in \triangle) .
$$

Here, $X_{s, t}^{2 ; i, j}$ stands for the $(i, j)$-component of $X_{s, t}^{2}$, etc. Obviously, a smooth rough path satisfies (3.4) and is an $\alpha$-Hölder weakly geometric rough path. The set of $\alpha$-Hölder weakly geometric rough paths is denoted by $G^{w} \Omega_{\alpha}^{H}\left(\mathbf{R}^{d}\right)$, which is a closed subset of $\Omega_{\alpha}^{H}\left(\mathbf{R}^{d}\right)$. In a similar way, $G^{w} \Omega_{\alpha, m}^{B}\left(\mathbf{R}^{d}\right)$ is defined. From (3.3) and (3.4), a weakly geometric rough path is a "difference" of a $G^{2}\left(\mathbf{R}^{d}\right)$-valued path.

Let $G \Omega_{\alpha}^{H}\left(\mathbf{R}^{d}\right)$ be the closure of the set of smooth rough paths, which is called the geometric rough path space with $\alpha$-Hölder norm. The geometric rough path space $G \Omega_{\alpha, m}^{B}\left(\mathbf{R}^{d}\right)$ with $(\alpha, m)$-Besov norm is similarly defined. Hence, we have the following inclusions:

$$
G \Omega_{\alpha}^{H}\left(\mathbf{R}^{d}\right) \subset G^{w} \Omega_{\alpha}^{H}\left(\mathbf{R}^{d}\right) \subset \Omega_{\alpha}^{H}\left(\mathbf{R}^{d}\right), \quad G \Omega_{\alpha, m}^{B}\left(\mathbf{R}^{d}\right) \subset G^{w} \Omega_{\alpha, m}^{B}\left(\mathbf{R}^{d}\right) \subset \Omega_{\alpha, m}^{B}\left(\mathbf{R}^{d}\right) .
$$

There is a natural correspondence between $G^{2}\left(\mathbf{R}^{d}\right)$-valued path spaces in the usual sense and weakly geometric rough path spaces. In order to explain that, we first introduce a distance $d$ on $G^{2}\left(\mathbf{R}^{d}\right)$. Let $\|\mathbf{a}\|$ be the Carnot-Caratheodory norm as in Chapter 7, 10. This is a homogeneous norm on $G^{2}\left(\mathbf{R}^{d}\right)$ with symmetry and 
subadditivity, too. Its explicit form is not needed in this paper. For our purpose, it is enough to keep in mind that there exists a constant $c>0$ such that

$$
\frac{1}{c}\|\mathbf{a}\| \leq\left|a_{1}\right|_{\mathbf{R}^{d}}+\sqrt{\left|a_{2}\right|_{\mathbf{R}^{d} \otimes \mathbf{R}^{d}}} \leq c\|\mathbf{a}\| \quad \text { for any } \mathbf{a}=\left(1, a_{1}, a_{2}\right) \in G^{2}\left(\mathbf{R}^{d}\right) .
$$

We set $d(\mathbf{a}, \mathbf{b})=\left\|\mathbf{a}^{-1} \otimes \mathbf{b}\right\|$. This defines a left-invariant distance on $G^{2}\left(\mathbf{R}^{d}\right)$, which induces the same topology as the relative one inherited from $T^{2}\left(\mathbf{R}^{d}\right)$.

If $\sup _{0<s<t \leq 1} d\left(g_{s}, g_{t}\right)|t-s|^{-\alpha}<\infty$, a $G^{2}\left(\mathbf{R}^{d}\right)$-valued continuous path $g$ is said to be $\alpha$-Hölder continuous. The set of such paths starting at $\mathbf{1}$ is denoted by $C_{\mathbf{1}}^{\alpha-H}\left([0,1], G^{2}\left(\mathbf{R}^{d}\right)\right)$. Similarly, $g$ is said to be $(\alpha, m)$-Besov if

$$
\iint_{0 \leq s<t \leq 1} d\left(g_{s}, g_{t}\right)^{m}|t-s|^{-(1+m \alpha)} d s d t<\infty
$$

The set of such paths starting at $\mathbf{1}$ is denoted by $C_{\mathbf{1}}^{\alpha, m-B}\left([0,1], G^{2}\left(\mathbf{R}^{d}\right)\right)$. For a weakly geometric rough path $X$, we can associate a $G^{2}\left(\mathbf{R}^{d}\right)$-valued continuous path $t \mapsto X_{0, t}$. This defines a natural bijection between $C_{1}^{\alpha-H}\left([0,1], G^{2}\left(\mathbf{R}^{d}\right)\right)$ and $G^{w} \Omega_{\alpha}^{H}\left(\mathbf{R}^{d}\right)$. Similarly, there is a natural bijection between $C_{\mathbf{1}}^{\alpha, m-B}\left([0,1], G^{2}\left(\mathbf{R}^{d}\right)\right)$ and $G^{w} \Omega_{m, \alpha}^{B}\left(\mathbf{R}^{d}\right)$. Through these bijections, we introduce distance functions on $C_{\mathbf{1}}^{\alpha-H}\left([0,1], G^{2}\left(\mathbf{R}^{d}\right)\right)$ and $C_{\mathbf{1}}^{m, \alpha-B}\left([0,1], G^{2}\left(\mathbf{R}^{d}\right)\right)$, respectively.

Proposition 3.1. Assume $1 / 3<\alpha<1 / 2, m \geq 2$, and $\alpha-1 / m>1 / 3$. Then, $G \Omega_{\alpha, m}^{B}\left(\mathbf{R}^{d}\right)$ is continuously embedded in $G \Omega_{\alpha-1 / m}^{H}\left(\mathbf{R}^{d}\right)$.

Proof. It is sufficient to show that $W \Omega_{\alpha, m}^{B}\left(\mathbf{R}^{d}\right) \cong C_{\mathbf{1}}^{\alpha, m-B}\left([0,1], G^{2}\left(\mathbf{R}^{d}\right)\right)$ is continuously embedded in $W \Omega_{\alpha-1 / m}^{H}\left(\mathbf{R}^{d}\right) \cong C_{\mathbf{1}}^{\left(\alpha-m^{-1}\right)-H}\left([0,1], G^{2}\left(\mathbf{R}^{d}\right)\right)$. The inclusion, which is called Besov-Hölder embedding, is shown in Corollary A.2, 10. Its continuity is shown in Proposition A.9, [10.

It is easy to see from Proposition 3.1 above that $G \Omega_{\alpha, m}^{B}\left(\mathbf{R}^{d}\right)$ is a complete separable metric space if $1 / 3<\alpha<1 / 2, m \geq 2$, and $\alpha-1 / m>1 / 3$.

Proposition 3.2. Assume $1 / 3<\alpha<\alpha^{\prime}<1 / 2, m \geq 2$, and $\alpha-1 / m>1 / 3$. Then, the injection $G \Omega_{\alpha^{\prime}, m}^{B}\left(\mathbf{R}^{d}\right) \hookrightarrow G \Omega_{\alpha, m}^{B}\left(\mathbf{R}^{d}\right)$ maps a bounded subset to a precompact subset.

Proof. Let $\{X(n)\}_{n=1}^{\infty}$ be any bounded sequence in $G \Omega_{\alpha^{\prime}, m}^{B}\left(\mathbf{R}^{d}\right)$. Define $g(n)_{t}=$ $X(n)_{0, t}$. Then, by Proposition 3.1. $\{g(n)\}_{n=1}^{\infty}$ is a bounded sequence in $C_{\mathbf{1}}^{\left(\alpha-m^{-1}\right)-H}\left([0,1], G^{2}\left(\mathbf{R}^{d}\right)\right)$. Hence, as functions of $t,\{g(n)\}_{n=1}^{\infty}$ is uniformly bounded and equicontinuous. By Ascoli-Arzela's theorem, there exists a subsequence, which is denoted by $\{g(n)\}$ again, which converges to $g \in C_{\mathbf{1}}\left([0,1], G^{2}\left(\mathbf{R}^{d}\right)\right)$ in sup-norm. Set $X_{s, t}=g_{s}^{-1} \otimes g_{t}$ for all $(s, t) \in \triangle$. It is sufficient to show that $\lim _{n \rightarrow \infty} X(n)=X$ in $(\alpha, m)$-Besov topology. First, note that

$$
\lim _{n \rightarrow \infty} \sup _{(s, t) \in \triangle}\left|X(n)_{s, t}^{j}-X_{s, t}^{j}\right|=0 \quad \text { for } j=1,2 .
$$


Set $r=\left(1+m \alpha^{\prime}\right) /(1+m \alpha)>1$ and let $r^{\prime}$ be its conjugate exponent. Then, by Hölder's inequality,

$$
\begin{aligned}
& \iint_{0 \leq s<t \leq 1} \frac{\left|X(n)_{s, t}^{1}-X\left(n^{\prime}\right)_{s, t}^{1}\right|^{m}}{|t-s|^{1+m \alpha}} d s d t \\
& \leq \sup _{(s, t) \in \triangle}\left|X(n)_{s, t}^{1}-X\left(n^{\prime}\right)_{s, t}^{1}\right|^{m(1-1 / r)} \iint_{0 \leq s<t \leq 1} \frac{\left|X(n)_{s, t}^{1}-X\left(n^{\prime}\right)_{s, t}^{1}\right|^{m / r}}{|t-s|^{1+m \alpha}} d s d t \\
& \leq \sup _{(s, t) \in \triangle}\left|X(n)_{s, t}^{1}-X\left(n^{\prime}\right)_{s, t}^{1}\right|^{m(1-1 / r)} \\
& \quad \times\left(\iint_{0 \leq s<t \leq 1} \frac{\left|X(n)_{s, t}^{1}-X\left(n^{\prime}\right)_{s, t}^{1}\right|^{m}}{|t-s|^{1+m \alpha^{\prime}}} d s d t\right)^{1 / r} \frac{1}{2^{1 / r^{\prime}}} \\
& \leq \text { const. } \times \sup _{(s, t) \in \triangle}\left|X(n)_{s, t}^{1}-X\left(n^{\prime}\right)_{s, t}^{1}\right|^{m(1-1 / r)} \rightarrow 0 \quad \text { as } n, n^{\prime} \rightarrow \infty .
\end{aligned}
$$

Here, we used that the $\left(\alpha^{\prime}, m\right)$-Besov norm of $X(n)$ is bounded. The limit in the Besov sense must coincide with $X^{1}$. The second level path can be dealt with in the same way.

\section{Preliminaries From QUASI-SURE ANALYSiS}

In this section we recall basics of Malliavin calculus and, in particular, of quasisure analysis. Generalized Wiener functionals (i.e., Watanabe distributions) and capacities associated with Gaussian Sobolev spaces play important roles in this paper. Analysis of these objects is called quasi-sure analysis.

4.1. Basics of Malliavin calculus. In this subsection, we recall the basic notions in Malliavin calculus, in particular, the theory of Gaussian Sobolev spaces. We mainly follow Sections 5.8-5.10, [14, or Shigekawa 25].

Let $(\mathcal{W}, \mathcal{H}, \mu)$ be an abstract Wiener space. As usual, $\mathcal{H}$ and $\mathcal{H}^{*}$ are identified through Riesz isometry. Let $D$ be the $\mathcal{H}$-derivative and $D^{*}$ be its dual. OrnsteinUhlenbeck operator is denoted by $L=-D^{*} D$. The first Wiener chaos associated with $h \in \mathcal{H}$ is denoted by $\langle h, w\rangle$. If $\langle h, \cdot\rangle \in \mathcal{W}^{*}$, then it coincides with $\mathcal{W}^{*}\langle h, w\rangle_{\mathcal{W}}$. A function $F: \mathcal{W} \rightarrow \mathbf{R}$ is called a real-valued polynomial if there exist $m \in$ $\mathbf{N}, h_{1}, \ldots, h_{m}$, and a real-valued polynomial $f$ of $m$-variables such that $F(w)=$ $f\left(\left\langle h_{1}, w\right\rangle, \ldots,\left\langle h_{m}, w\right\rangle\right)$. The set of all real-valued polynomials is denoted by $\mathcal{P}$. Let $\mathcal{K}$ be a real separable Hilbert space. $L^{q}(\mathcal{K})=L^{q}(\mathcal{W} ; \mathcal{K})$ denotes the $L^{q}$-space of $\mathcal{K}$-valued functions. A function $G: \mathcal{W} \rightarrow \mathcal{K}$ is called a $\mathcal{K}$-valued polynomial if there exist $m \in \mathbf{N}_{+}, F_{1}, \ldots, F_{m} \in \mathcal{P}$, and $v_{1}, \ldots, v_{m} \in \mathcal{K}$ such that $G(w)=$ $\sum_{j=1}^{m} F_{j}(w) v_{j}$. The set of all $\mathcal{K}$-valued polynomials is denoted by $\mathcal{P}(\mathcal{K})$.

For $q \in(1, \infty)$ and $r \in \mathbf{R}$, and $F \in \mathcal{P}(\mathcal{K})$, set $\|F\|_{q, r}=\left\|(I-L)^{-r / 2} F\right\|_{q}$. We define the Sobolev space $\mathbf{D}_{q, r}(\mathcal{K})$ to be the completion of $\mathcal{P}(\mathcal{K})$ with respect to this norm. When $\mathcal{K}=\mathbf{R}$, we simply write $L^{q}, \mathbf{D}_{q, r}$, etc. If $q \leq q^{\prime}$ and $r \leq r^{\prime}$, then $\|\cdot\|_{q, r} \leq\|\cdot\|_{q^{\prime}, r^{\prime}}$ and $\mathbf{D}_{q, r}(\mathcal{K}) \supset \mathbf{D}_{q^{\prime}, r^{\prime}}(\mathcal{K})$.

When $r=k \in \mathbf{N}$, then the Meyer equivalence holds (see 25] for example); there exists a positive constant $c_{q, r}$ such that

$$
c_{q, k}^{-1}\|F\|_{q, k} \leq\|F\|_{q}+\left\|D^{k} F\right\|_{q} \leq c_{q, k}\|F\|_{q, k} \quad \text { for all } F \in \mathbf{D}_{q, k}(\mathcal{K}) .
$$

Now we discuss the Wiener chaos. Let $\hat{\mathcal{C}}_{n}$ be the $L^{2}$-closure of real-valued polynomials of order $\leq n$. In particular, $\hat{\mathcal{C}}_{0}$ is the space of constant functions on 
$\mathcal{W} . \hat{\mathcal{C}}_{n}$ is called the inhomogeneous Wiener chaos of order $n$. Set $\mathcal{C}_{0}=\hat{\mathcal{C}}_{0}$ and $\mathcal{C}_{n}=\hat{\mathcal{C}}_{n} \cap \mathcal{C}_{n-1}^{\perp}$. This is called the homogeneous Wiener chaos of order $n$. Note that $\mathcal{C}_{1}=\{\langle h, \cdot\rangle \mid h \in \mathcal{H}\}$. It is known that $\mathcal{C}_{n}$ is the eigenspace of $-L$ in $L^{2}$ that corresponds to the eigenvalue $n$. Clearly, $\hat{\mathcal{C}}_{n}=\bigoplus_{k=0}^{n} \mathcal{C}_{k}$.

Proposition 4.1. For any $n \in \mathbf{N}, q \in[2, \infty)$ and $r \geq 0$, there exists a constant $M_{n, q, r} \geq 1$ such that

$$
M_{n, q, r}^{-1}\|F\|_{2} \leq\|F\|_{q, r} \leq M_{n, q, r}\|F\|_{2} \quad\left(F \in \hat{\mathcal{C}}_{n}\right) .
$$

In other words, restricted on $\hat{\mathcal{C}}_{n}$, all $(q, r)$-Sobolev norms are equivalent.

Proof. It is sufficient to show (4.1) for $\mathcal{C}_{n}$, instead of $\hat{\mathcal{C}}_{n}$. It is shown in Proposition 2.14 of [25] that all $L^{q}$-norms are equivalent on $\mathcal{C}_{n}$. Using the fact that $\mathcal{C}_{n}$ is an eigenspace of $-L$, we can easily see that all $(q, r)$-Sobolev norms are equivalent on $\mathcal{C}_{n}$.

Now we recall generalized Wiener functionals, which are also called Watanabe distributions. Set

$$
\mathbf{D}_{\infty}(\mathcal{K})=\bigcap_{1<q<\infty, r \in \mathbf{R}} \mathbf{D}_{q, r}(\mathcal{K}) \quad \text { and } \quad \mathbf{D}_{-\infty}(\mathcal{K})=\bigcup_{1<q<\infty, r \in \mathbf{R}} \mathbf{D}_{q, r}(\mathcal{K}) .
$$

Those are called the space of test functions and the space of generalized Wiener functionals, respectively. We also use the following spaces of (generalized) Wiener functionals:

$$
\tilde{\mathbf{D}}_{\infty}(\mathcal{K})=\bigcap_{k=1}^{\infty} \bigcup_{1<q<\infty} \mathbf{D}_{q, k}(\mathcal{K}) \text { and } \quad \tilde{\mathbf{D}}_{-\infty}(\mathcal{K})=\bigcup_{k=1}^{\infty} \bigcap_{1<q<\infty} \mathbf{D}_{q,-k}(\mathcal{K}) .
$$

In other words, $F \in \tilde{\mathbf{D}}_{\infty}(\mathcal{K})$ is equivalent to that, for any $k \geq 0$, there exists $q=q(k)>1$ such that $F \in \mathbf{D}_{q, k}(\mathcal{K})$.

Let $F=\left(F^{1}, \ldots, F^{n}\right) \in \mathbf{D}_{\infty}\left(\mathbf{R}^{n}\right)$. The Malliavin covariance matrix is defined by $\left(\left\langle D F^{i}(w), D F^{j}(w)\right\rangle_{\mathcal{H}}\right)_{1 \leq i, j \leq n}$. We say that $F$ is non-degenerate in the sense of Malliavin if $\operatorname{det}\left(\left\langle D F^{i}(w), D F^{j}(w)\right\rangle_{\mathcal{H}}\right)_{1 \leq i, j \leq n}^{-1}$ is in $L^{q}$ for all $1<q<\infty$. This non-degeneracy is very important in Malliavin calculus. For example, such a nondegenerate $F$ can be composed with a Schwartz distribution $\phi$ defined on $\mathbf{R}^{n}$ and $\phi \circ F$ becomes a generalized Wiener functional.

We introduce seminorms on the space of tempered distributions on $\mathbf{R}^{n}$. For $k \in \mathbf{Z}$ and a real-valued, rapidly decreasing, smooth function $\phi$ of Schwartz class $\mathcal{S}\left(\mathbf{R}^{n}\right)$ on $\mathbf{R}^{n}$, we define $\|\phi\|_{2 k}=\left\|\left(1+|\cdot|^{2}-\triangle / 2\right)^{k} \phi\right\|_{\infty}$. Set $\mathcal{S}_{2 k}\left(\mathbf{R}^{n}\right)$ to be the completion of $\mathcal{S}\left(\mathbf{R}^{n}\right)$ with respect to this norm. Then $\mathcal{S}\left(\mathbf{R}^{n}\right)=\bigcap_{k>0} \mathcal{S}_{2 k}\left(\mathbf{R}^{n}\right)$, which is a Fréchet space. The dual space $\mathcal{S}^{\prime}\left(\mathbf{R}^{n}\right)=\bigcup_{k>0} \mathcal{S}_{-2 k}\left(\mathbf{R}^{n}\right)$ is called the Schwartz space of tempered distributions.

S. Watanabe proved that, if a Wiener functional $F$ is non-degenerate, then the pullback map $\phi \mapsto \phi \circ F$ extends to a continuous linear map between two distribution spaces. The following is borrowed from pp. 378-379 in Ikeda and Watanabe [14.

Theorem 4.2. Let $F=\left(F^{1}, \ldots, F^{n}\right) \in \mathbf{D}_{\infty}\left(\mathbf{R}^{n}\right)$ be non-degenerate in the sense of Malliavin. Then, for any $1<q<\infty$ and $k=0,1,2, \ldots$, there exists a positive constant $C=C(q, k, F)$ such that

$$
\|\phi \circ F\|_{q,-2 k} \leq C\|\phi\|_{-2 k} \quad\left(\phi \in \mathcal{S}\left(\mathbf{R}^{n}\right)\right)
$$


holds. Therefore, the map $\phi \mapsto \phi \circ F$ extends uniquely to a continuous linear map $\mathcal{S}_{-2 k}\left(\mathbf{R}^{n}\right) \ni T \mapsto T \circ F \in \mathbf{D}_{q,-2 k}$. In particular, $T \circ F \in \bigcup_{k=1}^{\infty} \bigcap_{1<q<\infty} \mathbf{D}_{q,-2 k}=$ $\tilde{\mathbf{D}}_{-\infty}$.

We recall an integration by parts formula in the context of Malliavin calculus (see p. 377, [14]). For $F=\left(F^{1}, \ldots, F^{n}\right) \in \mathbf{D}_{\infty}\left(\mathbf{R}^{n}\right)$, we denote by $\tau^{i j}(w)=$ $\left\langle D F^{i}(w), D F^{j}(w)\right\rangle_{\mathcal{H}}$ the $(i, j)$-component of Malliavin covariance matrix. We denote by $\gamma^{i j}(w)$ the $(i, j)$-component of the inverse matrix $\tau^{-1}$. Note that $\tau^{i j} \in \mathbf{D}_{\infty}$ and $D \gamma^{i j}=\sum_{k, l} \gamma^{i k}\left(D \tau^{k l}\right) \gamma^{l j}$. Hence, derivatives of $\gamma^{i j}$ can be written in terms of $\gamma^{i j}$ 's and the derivatives of $\tau^{i j}$ 's. Suppose $G \in \mathbf{D}_{\infty}$ and $T \in \mathcal{S}^{\prime}\left(\mathbf{R}^{n}\right)$. Then, the following integration by parts holds if $F$ is non-degenerate:

$$
\mathbb{E}\left[\left(\partial_{i} T \circ F\right) \cdot G\right]=\mathbb{E}\left[(T \circ F) \cdot \Phi_{i}(\cdot ; G)\right]
$$

where $\Phi_{i}(\cdot ; G) \in \mathbf{D}_{\infty}$ is given by

$$
\begin{aligned}
\Phi_{i}(w ; G)=-\sum_{j=1}^{d}\{ & -\sum_{k, l=1}^{d} G(w) \gamma^{i k}(w) \gamma^{j l}(w)\left\langle D \tau^{k l}(w), D F^{j}(w)\right\rangle_{\mathcal{H}} \\
& \left.+\gamma^{i j}(w)\left\langle D G(w), D F^{j}(w)\right\rangle_{\mathcal{H}}+\gamma^{i j}(w) G(w) L F^{j}(w)\right\} .
\end{aligned}
$$

Note that the expectations in (4.2) are in fact the pairing of $\tilde{\mathbf{D}}_{-\infty}$ and $\tilde{\mathbf{D}}_{\infty}$.

4.2. Basics of capacities. In this subsection, we recall the definition and basic properties of the capacity associated with the Sobolev space $\mathbf{D}_{q, r}$ for $1<q<\infty$ and $r \in \mathbf{N}$.

The contents of the subsection are borrowed from Chapter 9, Malliavin 21. In this book, they work on a particular Gaussian space, namely $\mathbf{R}^{\infty}$ equipped with countable product of one-dimensional standard normal distribution. But, the results in 21, at least the ones we will use in this paper, hold true on any abstract Wiener space. In some literature, a slightly different definition of capacities is used (see [26] for instance).

Let $(\mathcal{W}, \mathcal{H}, \mu)$ be an abstract Wiener space. We keep the same notation as in the previous subsection. Throughout this subsection, we set $1<q<\infty$ and $r \in \mathbf{N}$. For an open subset $O \subset \mathcal{W}$, we set

$$
\mathrm{c}_{q, r}(O)=\inf \left\{\|\phi\|_{q, r} \mid \phi(w) \geq 1 \text { a.e. on } O \text { and } \phi(w) \geq 0 \text { a.e. on } \mathcal{W}\right\} \text {. }
$$

For any subset $A \subset \mathcal{W}$, which is not necessarily open, we define

$$
\mathrm{c}_{q, r}(A)=\inf \left\{\mathrm{c}_{q, r}(O) \mid A \subset O \text { and } O \text { is open }\right\} .
$$

Since $(q, r)$-norm is increasing in both $q$ and $r$, so is $c_{q, r}(A)$. We say that a property $\pi=\pi_{w}$ holds $(q, r)$-quasi-everywhere if $\mathrm{c}_{q, r}\left(\left\{w \mid \pi_{w}\right.\right.$ does not hold $\left.\}\right)=0$. We say that a property $\pi=\pi_{w}$ holds quasi-surely if it holds $(q, r)$-quasi-everywhere for all $q \in(1, \infty)$ and $r \in \mathbf{N}$. A subset $A$ is called slim if $\mathrm{c}_{q, r}(A)=0$ for all $q \in(1, \infty)$ and $r \in \mathbf{N}$.

Now let us discuss quasi-continuity. A function $\phi$ from $\mathcal{W}$ to $\mathbf{R}$ (or to a metric space) is said to be $(q, r)$-quasi-continuous if, for any $\varepsilon>0$, there exists an open set $O_{\varepsilon}$ such that $\mathrm{c}_{q, r}\left(O_{\varepsilon}\right)<\varepsilon$ and the restriction $\left.\phi\right|_{O_{\varepsilon}^{c}}$ is continuous. A function $\phi$ from $\mathcal{W}$ to $\mathbf{R}$ (or to a metric space) is said to be $\infty$-quasi-continuous if it is $(q, r)$-quasicontinuous for all $q$ and $r$. This is equivalent to that there is a decreasing sequence of open subsets $O_{n}$ such that $\lim _{n \rightarrow \infty} \mathrm{c}_{n, n}\left(O_{n}\right)=0$ and $\left.\phi\right|_{O_{n}^{c}}$ is continuous. (In 
this paragraph, the set $O_{\varepsilon}$ or $O_{n}$ actually need not be open, since any subset can be approximated in terms of capacity by an open subset from outside.)

For a measurable function $\psi$, we say $\psi^{*}$ is a $(q, r)$-redefinition of $\psi$ if $\psi^{*}$ is $(q, r)$-quasi-continuous and $\psi=\psi^{*}$ a.s. $\left(\psi^{*}\right.$ is also called $(q, r)$-quasi continuous modification). Note that $(q, r)$-redefinition is essentially unique when it exists. It is shown in Theorem 2.3.3 of [21] that any $\phi \in \mathbf{D}_{q, r}$ admits a $(q, r)$-redefinition. Similarly, for a measurable function $\psi$, we say $\psi^{*}$ is a $\infty$-redefinition of $\psi$ if $\psi^{*}$ is $\infty$-quasi-continuous and $\psi=\psi^{*}$ a.s. It is shown in Subsection 2.4 of [21] that any $\phi \in \mathbf{D}_{\infty}$ admits an (essentially unique) $\infty$-redefinition.

Now we give two useful basic lemmas without proofs for later use. One is BorelCantelli's lemma (Corollary 1.2.4, 21]) and the other is Chebyshev's lemma (Theorem 2.2, 21]).

Lemma 4.3. Let $1<q<\infty$ and $r \in \mathbf{N}$. Assume that $A_{k} \subset \mathcal{W}(k \in \mathbf{N})$ satisfy that $\sum_{k} \mathrm{c}_{q, r}\left(A_{k}\right)<\infty$. Then, $\mathrm{c}_{q, r}\left(\lim \sup _{k \rightarrow \infty} A_{k}\right)=0$.

Lemma 4.4. For any $1<q<\infty$ and $r \in \mathbf{N}$, there exists a positive constant $M_{q, r}$ such that, for any $\phi \in \mathbf{D}_{q, r}$ and any $R>0$, we have

$$
\mathrm{c}_{q, r}\left(\left\{w \mid \phi^{*}(w)>R\right\}\right) \leq R^{-1} M_{q, r}\|\phi\|_{q, r} .
$$

In the finite-dimensional calculus, it is known that a positive Schwartz distribution is a measure. An analogous fact is true in Malliavin calculus, too. It is called Sugita's theorem (Theorem 3.0, 21] or Sugita [26]) and will play an important role in the sequel.

Proposition 4.5. Let $l \in \mathbf{D}_{-\infty}$ be a positive Watanabe distribution, that is, it satisfies $\mathbf{D}_{-\infty}\langle l, f\rangle_{\mathbf{D}_{\infty}}=\mathbb{E}[l \cdot f] \geq 0$ for any $f \in \mathbf{D}_{\infty}$ such that $f>0$ a.s. Then, there exists a unique positive Borel measure $\theta$ of finite total mass such that

$$
\langle l, g\rangle=\mathbb{E}[l \cdot g]=\int_{\mathcal{W}} g^{*}(w) d \theta(w) \quad\left(g \in \mathbf{D}_{\infty}\right) .
$$

Furthermore, $\theta$ does not charge a slim set. (Hence, any choice of $\infty$-redefinition $g^{*}$ will do.)

It is easy to see that, if $l \in \mathbf{D}_{q^{\prime},-r}$ with $1 / q+1 / q^{\prime}=1$, then $\theta(O) \leq \mathrm{c}_{q, r}(O)\|l\|_{q^{\prime},-r}$ for any open set $O$. (Note that $O$ need not be open here again.)

Let us discuss the equilibrium potential. Given a Borel subset $A \subset \mathcal{W}$, we define

$$
\mathcal{F}_{q, r}(A)=\left\{u \in \mathbf{D}_{q, r} \mid u^{*} \geq 1(q, r) \text {-quasi-everywhere on } A\right\} .
$$

This is a closed convex subset of $\mathbf{D}_{q, r}$ and has a unique element $\phi_{A} \in \mathcal{F}_{q, r}(A)$, which minimizes $(q, r)$-Sobolv norm. We call $\phi_{A}$ the equilibrium potential of $A$. Then, Theorem 4.4, 21] states that,

$$
\mathrm{c}_{q, r}(A)=\left\|\phi_{A}\right\|_{q, r} \leq\|u\|_{q, r} \quad\left(u \in \mathcal{F}_{q, r}(A)\right) .
$$

\section{QuASI-SURE EXISTENCE OF A BROWNIAN ROUGH PATH}

In this section, we recall that Brownian motion admits a natural lift quasi-surely via the dyadic partitions. This fact was proved by three authors independently, Higuchi [12], Inahama [15], and Watanabe [30]. Among them, Higuchi's method seems best. Higuchi's master thesis is in Japanense and probably unavailable outside Japan. However, Section 3 of Aida's recent paper [1] is essentially the same. So we will follow [1,12, in which a slightly different Besov norm is used. 
From now on, we denote by $(\mathcal{W}, \mathcal{H}, \mu)$ the $d$-dimensional classical Wiener space. That is, $\mathcal{W}=C_{0}\left([0,1], \mathbf{R}^{d}\right)$ with the sup-norm, $\mathcal{H}$ is the Cameron-Martin space, and $\mu$ is the usual $d$-dimensional Wiener measure. For $w \in \mathcal{W}$ and $k \in \mathbf{N}, w(k) \in$ $C_{0}^{1-H}\left([0,1], \mathbf{R}^{d}\right)$ denotes the $m$ th dyadic polygonal approximation associated with the partition $\left\{l 2^{-k} \mid 0 \leq l \leq 2^{k}\right\}$ of $[0,1]$. We denote by $W(k):=S_{2}(w(k))$ the natural lift of $w(k)$.

For $\alpha, m$ such that $4 m \geq 2,1 / 3<\alpha<1 / 2$, and $\alpha-1 /(4 m)>1 / 3$, we set

$$
\mathcal{Z}_{\alpha, 4 m}=\left\{w \in \mathcal{W} \mid\{W(k)\}_{k=1}^{\infty} \text { is Cauchy in } G \Omega_{\alpha, 4 m}^{B}\left(\mathbf{R}^{d}\right)\right\} .
$$

Slightly abusing the notation, we denote $\lim _{k \rightarrow \infty} W(k)$ by $W$ or $S_{2}(w)$ for $w \in$ $\mathcal{Z}_{\alpha, 4 m}$. If $w \notin \mathcal{Z}_{\alpha, 4 m}, S_{2}(w)$ is not defined. (So, as a map from $\mathcal{W}, S_{2}$ 's definition depends on $\alpha, m$.) The aim of this section is to prove that, for sufficiently large $m \in \mathbf{N}, \mathcal{Z}_{\alpha, 4 m}^{c}$ is a slim set.

Some basic properties of $\mathcal{Z}_{\alpha, 4 m}$ are shown in the following lemma. We can see from this that we may write $c W(c \in \mathbf{R})$ without ambiguity.

Lemma 5.1. Let $\alpha, m$ and $\mathcal{Z}_{\alpha, 4 m}$ be as above. Then, we have the following (i)-(iii):

(i) $\mathcal{H} \subset \mathcal{Z}_{\alpha, 4 m}$.

(ii) For any $c \in \mathbf{R}$ and $w \in \mathcal{Z}_{\alpha, 4 m}, S_{2}(c w)=c S_{2}(w)$. In particular, $c \mathcal{Z}_{\alpha, 4 m}=$ $\mathcal{Z}_{\alpha, 4 m}$ if $c \neq 0$.

(iii) Assume in addition that $2 m-4 m \alpha-1=4 m(1 / 2-\alpha)-1>0$. Then, for any $h \in \mathcal{H}$ and $w \in \mathcal{Z}_{\alpha, 4 m}, S_{2}(w+h)=T_{h}\left(S_{2}(w)\right)$. Here, the right hand side stands for the Young translation of $S_{2}(w)$ by $h$. In particular, $T_{h}\left(\mathcal{Z}_{\alpha, 4 m}\right)=\mathcal{Z}_{\alpha, 4 m}$.

Proof. Throughout this proof, the constant $C>0$ may change from line to line. (ii) is trivial. Note that $\|h\|_{1 / 2-H} \leq\|h\|_{\mathcal{H}}$ and that, for any $h,\|h(k) \rightarrow h\|_{\mathcal{H}} \rightarrow 0$ as $k \rightarrow \infty$. Then, (i) is immediate from these.

We now prove (iii). The only non-trivial part is boundedness of the "cross terms" of the second level path as bilinear functionals in $X^{1}$ and $h$. For $x \in$ $C_{0}^{\alpha, 4 m-B}\left([0,1], \mathbf{R}^{d}\right)$ and $h \in \mathcal{H}$, consider

$$
J[x, h]_{s, t}:=\int_{s}^{t}\left(x_{u}-x_{s}\right) \otimes d h_{u} .
$$

Then, we can easily see from (3.1) that

$$
\begin{aligned}
\left|J[x, h]_{s, t}\right|^{2} & \leq\left(\int_{s}^{t}\left|x_{u}-x_{s} \| h_{u}^{\prime}\right| d u\right)^{2} \leq\|h\|_{\mathcal{H}}^{2} \int_{s}^{t}\left|x_{u}-x_{s}\right|^{2} d u \\
& \leq C\|h\|_{\mathcal{H}}^{2}\|x\|_{\alpha, 4 m-B}^{2} \int_{s}^{t}(u-s)^{2(\alpha-1 / 4 m)} d u \\
& \leq C\|h\|_{\mathcal{H}}^{2}\|x\|_{\alpha, 4 m-B}^{2}(t-s)^{2(\alpha-1 / 4 m)+1} .
\end{aligned}
$$

Then, we have

$$
\begin{aligned}
\|J[x, h]\|_{2 \alpha, 2 m-B}^{2 m} & \leq C\|h\|_{\mathcal{H}}^{2 m}\|x\|_{\alpha, 4 m-B}^{2 m} \iint_{0 \leq s \leq t \leq 1} \frac{(t-s)^{2 m(\alpha-1 / 4 m)+m}}{(t-s)^{1+4 m \alpha}} d s d t \\
& \leq C\|h\|_{\mathcal{H}}^{2 m}\|x\|_{\alpha, 4 m-B}^{2 m} \iint_{0 \leq s \leq t \leq 1} \frac{1}{(t-s)^{1+2 m \alpha-m+1 / 2}} d s d t \\
& \leq C\|h\|_{\mathcal{H}}^{2 m}\|x\|_{\alpha, 4 m-B}^{2 m} .
\end{aligned}
$$

In the same way, $J[h, x]$ can be estimated. The rest is routine and we omit it. 
Take any $\varepsilon>0$ and fix it. We set

$$
\mathcal{A}_{\alpha, 4 m}^{k}=\left\{w \in \mathcal{W} \mid\|w(k+1)-w(k)\|_{\alpha, 4 m-B}^{4 m} \geq k^{-(4 m+\varepsilon)}\right\} .
$$

Note that $\|w(k+1)-w(k)\|_{\alpha, 4 m-B}^{4 m}$ is in the inhomogeneous Wiener chaos $\hat{\mathcal{C}}_{4 m}$ if $m \in \mathbf{N}$. We can estimate the capacities of $\mathcal{A}_{\alpha, 4 m}^{k}$ as follows.

Lemma 5.2. As before, let $m \in \mathbf{N}_{+}, 1 / 3<\alpha<1 / 2$, and $\alpha-(1 / 4 m)>1 / 3$. Furthermore, we assume that $4 m-8 m \alpha-1=8 m(1 / 2-\alpha)-1>0$. Then, for any $1<q<\infty$ and $r \in \mathbf{N}$,

$$
\sum_{k=1}^{\infty} \mathrm{c}_{q, r}\left(\mathcal{A}_{\alpha, 4 m}^{k}\right) \leq M_{q, r} \sum_{k=1}^{\infty} k^{4 m+\varepsilon}\|\| w(k+1)-w(k)\left\|_{\alpha, 4 m-B}^{4 m}\right\|_{q, r}<\infty .
$$

Here, $M_{q, r}>0$ is the constant given in Lemma 4.4. As a result, $\lim _{\sup } \sup _{k \rightarrow \infty} \mathcal{A}_{\alpha, 4 m}^{k}$ is slim, which implies quasi-sure convergence of $\left\{W(k)^{1}\right\}_{k}$.

Proof. The left inequality in (5.2) is immediate from Lemma 4.4. First we will prove the summability for $L^{2}$-norm, instead of $\mathbf{D}_{q, r}$-norm.

We set $z(k)=w(k+1)-w(k)$ and $Z(k)_{s, t}^{1}=z(k)_{t}-z(k)_{s}$ for simplicity. It can be written explicitly as follows:

$$
\begin{aligned}
z(k)_{t}=2^{k}\{(t & \left.\left.-\frac{j-1}{2^{k}}\right) \wedge\left(\frac{j}{2^{k}}-t\right)\right\} \\
& \times\left(2 w\left(\frac{2 j-1}{2^{k+1}}\right)-w\left(\frac{j-1}{2^{k}}\right)-w\left(\frac{j}{2^{k}}\right)\right) \quad \text { if } \frac{j-1}{2^{k}} \leq t \leq \frac{j}{2^{k}} .
\end{aligned}
$$

Note that $\|z(k)\|_{\alpha, 4 m-B}^{4 m} \in \hat{\mathcal{C}}_{4 m}$. A straightforward computation yields:

$$
\mathbb{E}\left[\left|Z(k)_{s, t}^{1}\right|^{2}\right] \leq C_{d}\left(\frac{1}{2^{k}} \wedge(t-s)\right), \quad(s, t) \in \triangle,
$$

for some constant $C_{d}>0$, which depends only on $d$. Then, we have

$$
\begin{aligned}
\|\| z(k)\left\|_{\alpha, 4 m-B}^{4 m}\right\|_{2}^{2} & \leq \mathbb{E}\left[\left(\iint_{0 \leq s \leq t \leq 1} \frac{\left|Z(k)_{s, t}^{1}\right|^{4 m}}{(t-s)^{1+4 m \alpha}} d s d t\right)^{2}\right] \\
& \leq \frac{1}{2} \iint_{0 \leq s \leq t \leq 1} \frac{\mathbb{E}\left[\left|Z(k)_{s, t}^{1}\right|^{8 m}\right]}{(t-s)^{2+8 m \alpha}} d s d t \\
& \leq C_{m, d} \iint_{0 \leq s \leq t \leq 1} \frac{\left(2^{-k}\right)^{4 m} \wedge(t-s)^{4 m}}{(t-s)^{2+8 m \alpha}} d s d t .
\end{aligned}
$$

Here, we used Schwarz's inequality and Proposition 4.1 for $Z(k)_{s, t}^{1} \in \mathcal{C}_{1}$.

Now use the following well-known inequality:

$$
\iint_{0 \leq s \leq t \leq 1} \frac{\eta \wedge(t-s)^{a}}{(t-s)^{b}} d s d t \leq \frac{a \eta^{(a-b+1) / a}}{(a-b+1)(b-1)} \quad(0<\eta \leq 1, b>1, a>b-1) .
$$

To check this formula, just change variables by $u=s, v=t-s$. Then, the domain of integral becomes $\{0<u<1,0<v<1, u+v<1\}$. The rest is easy.

Observe that, if $4 m-8 m \alpha-1=8 m(1 / 2-\alpha)-1>0$, we can use (5.6). We have

$$
\|\| z(k)\left\|_{\alpha, 4 m-B}^{4 m}\right\|_{2} \leq C_{\alpha, m, d}\left(\frac{1}{2^{(4 m-8 m \alpha-1) / 2}}\right)^{k} .
$$


This proves the summability for $L^{2}$-norm. As for $\mathbf{D}_{q, r}$-norm, we can easily see from Proposition 4.1 that

$$
\begin{aligned}
\sum_{k=1}^{\infty} k^{4 m+\varepsilon}\|\| z(k)\left\|_{\alpha, 4 m-B}^{4 m}\right\|_{q, r} & \leq M_{4 m, q, r} \sum_{k=1}^{\infty} k^{4 m+\varepsilon}\|\| z(k)\left\|_{\alpha, 4 m-B}^{4 m}\right\|_{2} \\
& \leq C_{q, r, \alpha, m, d} \sum_{k=1}^{\infty} k^{4 m+\varepsilon}\left(\frac{1}{2^{(4 m-8 m \alpha-1) / 2}}\right)^{k}<\infty .
\end{aligned}
$$

Here, $c_{q, r, \alpha, m, d}$ is a positive constant which depends only on $q, r, \alpha, m, d$.

It is immediate from Lemma 4.3 that $\lim \sup _{k \rightarrow \infty} \mathcal{A}_{\alpha, 4 m}^{k}$ is slim. Note that $w \in\left(\lim \sup _{k \rightarrow \infty} \mathcal{A}_{\alpha, 4 m}^{k}\right)^{c}=\bigcup_{N=1}^{\infty} \bigcap_{k=N}^{\infty}\left(\mathcal{A}_{\alpha, 4 m}^{k}\right)^{c}$ implies that $\{w(k)\}=\left\{W(k)^{1}\right\}$ is convergent in $(\alpha, m)$-Besov norm.

Next, let us consider the second level paths. For $\mathbf{R}^{d}$-valued continuous paths $x$ and $y$, we define $J[x, y]: \triangle \rightarrow\left(\mathbf{R}^{d}\right)^{\otimes 2}$ by

$$
J[x, y]_{s, t}=\int_{s}^{t}\left(x_{u}-x_{s}\right) \otimes d y_{u},
$$

whenever the integral on the right hand side can be defined. Note that the following equality holds:

$$
\begin{aligned}
J[x, x]_{s, t}-J[y, y]_{s, t}=J[ & x-y, x-y]_{s, t}+J[x-y, y]_{s, t} \\
& -J[x-y, y]_{s, t}^{*}+Y_{s, t}^{1} \otimes\left(X_{s, t}^{1}-Y_{s, t}^{1}\right) .
\end{aligned}
$$

Here, $*$ stands for the linear isometry of $\left(\mathbf{R}^{d}\right)^{\otimes 2}$ defined by $(\xi \otimes \eta)^{*}=\eta \otimes \xi$ for all $\xi, \eta \in \mathbf{R}^{d}$. Indeed, we can easily see that

$$
J[x-y, x-y]_{s, t}-J[x, x]_{s, t}+J[y, y]_{s, t}=-J[x-y, y]_{s, t}-J[y, x-y]_{s, t}
$$

and, from integration by parts, that

$$
J[y, x-y]_{s, t}=Y_{s, t}^{1} \otimes\left(X_{s, t}^{1}-Y_{s, t}^{1}\right)-\int_{s}^{t} d y_{u} \otimes\left(X_{s, u}^{1}-Y_{s, u}^{1}\right) .
$$

Thus, we have verified (5.8).

With (5.8) in hand, we naturally consider the following subsets in $\mathcal{W}$.

$$
\begin{aligned}
& \mathcal{B}(1)_{2 \alpha, 2 m}^{k}=\left\{w \in \mathcal{W} \mid\|J[z(k), z(k)]\|_{2 \alpha, 2 m-B}^{2 m} \geq k^{-(2 m+\varepsilon)}\right\}, \\
& \mathcal{B}(2)_{2 \alpha, 2 m}^{k}=\left\{w \in \mathcal{W} \mid\|J[z(k), w(k)]\|_{2 \alpha, 2 m-B}^{2 m} \geq k^{-(2 m+\varepsilon)}\right\} \\
& \mathcal{B}(3)_{2 \alpha, 2 m}^{k}=\left\{w \in \mathcal{W} \mid\left\|W(k)^{1} \otimes Z(k)^{1}\right\|_{2 \alpha, 2 m-B}^{2 m} \geq k^{-(2 m+\varepsilon)}\right\},
\end{aligned}
$$

where we set $z(k)=w(k+1)-w(k)$ as before.

Lemma 5.3. As before, let $m \in \mathbf{N}_{+}, 1 / 3<\alpha<1 / 2$, and $\alpha-(1 / 4 m)>1 / 3$. Furthermore, we assume that $4 m-8 m \alpha-1=8 m(1 / 2-\alpha)-1>0$. Then, for any 
$1<q<\infty$ and $r \in \mathbf{N}$

$$
\begin{aligned}
& \text { (5.12) } \sum_{k=1}^{\infty} \mathrm{c}_{q, r}\left(\mathcal{B}(1)_{2 \alpha, 2 m}^{k}\right) \leq M_{q, r} \sum_{k=1}^{\infty} k^{2 m+\varepsilon}\|\| J[z(k), z(k)]\left\|_{2 \alpha, 2 m-B}^{2 m}\right\|_{q, r}<\infty, \\
& \text { (5.13) } \sum_{k=1}^{\infty} \mathrm{c}_{q, r}\left(\mathcal{B}(2)_{2 \alpha, 2 m}^{k}\right) \leq M_{q, r} \sum_{k=1}^{\infty} k^{2 m+\varepsilon}\|\| J[z(k), w(k)]\left\|_{2 \alpha, 2 m-B}^{2 m}\right\|_{q, r}<\infty, \\
& \text { (5.14) } \sum_{k=1}^{\infty} \mathrm{c}_{q, r}\left(\mathcal{B}(3)_{2 \alpha, 2 m}^{k}\right) \leq M_{q, r} \sum_{k=1}^{\infty} k^{2 m+\varepsilon}\|\| W(k)^{1} \otimes Z(k)^{1}\left\|_{2 \alpha, 2 m-B}^{2 m}\right\|_{q, r}<\infty .
\end{aligned}
$$

Here, $M_{q, r}>0$ is the constant given in Lemma 4.4 above. As a result, $\lim \sup _{k \rightarrow \infty} \mathcal{B}(i)_{2 \alpha, 2 m}^{k}$ is slim for all $i=1,2,3$, which implies quasi-sure convergence of $\left\{W(k)^{2}\right\}_{k}$.

Proof. Substituting $x=w(k+1)$ and $y=w(k)$ in (5.8), we have

$W(k+1)^{2}-W(k)^{2}=J[z(k), z(k)]+J[z(k), w(k)]-J[z(k), w(k)]^{*}+W(k)^{1} \otimes Z(k)^{1}$.

If $w \in \bigcap_{1 \leq i \leq 3}\left(\lim \sup _{k \rightarrow \infty} \mathcal{B}(i)_{\alpha, m}^{k}\right)^{c}$, then $\left\{W(k)^{2}\right\}_{k=1}^{\infty}$ is a Cauchy sequence in $(2 \alpha, 2 m)$-Besov norm. The left inequalities in (5.12) -(5.14) are obvious from Lemma 4.4 of Chebyshev type. By Lemma4.3 of Borel-Cantelli type, summability in (5.12)(5.14) implies that $\lim \sup _{k \rightarrow \infty} \mathcal{B}(i)_{\alpha, m}^{k}$ are slim for all $i$.

Hence, it is sufficient to show that the sums on the right hand sides of (5.12) (5.14) converge. First, we consider (5.12). It is not difficult to see that, for a positive constant $C_{d}$,

$$
\mathbb{E}\left[\left|J[z(k), z(k)]_{s, t}\right|^{2}\right] \leq C_{d}\left(\frac{1}{2^{2 k}} \wedge(t-s)^{2}\right), \quad(s, t) \in \triangle .
$$

A rough sketch of the proof of this estimate is as follows. Suppose that $(j-1) / 2^{k} \leq$ $s \leq j / 2^{k}$ and $(l-1) / 2^{k} \leq t \leq l / 2^{k}$ with $j \leq l$. If $j=l$, then we have from (5.3) that

$$
\begin{aligned}
J[z(k), z(k)]_{s, t}=\frac{2^{2 k}}{2}\{( & \left.\left.t-\frac{j-1}{2^{k}}\right) \wedge\left(\frac{j}{2^{k}}-t\right)-\left(s-\frac{j-1}{2^{k}}\right) \wedge\left(\frac{j}{2^{k}}-s\right)\right\}^{2} \\
& \times\left(2 w\left(\frac{2 j-1}{2^{k+1}}\right)-w\left(\frac{j-1}{2^{k}}\right)-w\left(\frac{j}{2^{k}}\right)\right)^{\otimes 2} .
\end{aligned}
$$

In this case, (5.15) is easy. If $j<l$, we use Chen's identity to obtain

$$
\begin{aligned}
J[z(k), z(k)]_{s, t}= & J[z(k), z(k)]_{s, j / 2^{k}}+J[z(k), z(k)]_{(l-1) / 2^{k}, t} \\
& +Z(k)_{s, j / 2^{k}}^{1} \otimes Z(k)_{(l-1) / 2^{k}, t}^{1} .
\end{aligned}
$$

In the same way as above, we can estimate the right hand side. Thus, we have shown (5.15).

With (5.15) in hand, we can calculate in the same way as in (5.5) to obtain

$$
\|\| J[z(k), z(k)]\left\|_{2 \alpha, 2 m-B}^{4 m}\right\|_{2} \leq C_{\alpha, m, d}\left(\frac{1}{2^{(4 m-8 m \alpha-1) / 2}}\right)^{k} .
$$

From this and Proposition 4.1. we can easily see that, if $4 m-8 m \alpha-1>0$, the sum in (5.12) converges.

Next, we consider (5.14). In this case we have

$$
\mathbb{E}\left[\left|W(k)_{s, t}^{1} \otimes Z(k)_{s, t}^{1}\right|^{2}\right] \leq C_{d}\left(\frac{1}{2^{k}} \wedge(t-s)^{2}\right), \quad(s, t) \in \triangle,
$$


for some positive constant $C_{d}$. From (5.4) and an estimate that $\mathbb{E}\left[\left|W(k)_{s, t}^{1}\right|^{2}\right] \leq$ $d(t-s)$ for all $k$, we can easily show (5.16).

From this and Proposition 4.1 for $W(k)_{s, t}^{1} \otimes Z(k)_{s, t}^{1} \in \hat{\mathcal{C}}_{2}$, we have

$$
\begin{aligned}
\|\| W(k)^{1} \otimes Z(k)\left\|_{2 \alpha, 2 m-B}^{2 m}\right\|_{2}^{2} & \leq \mathbb{E}\left[\left(\iint_{0 \leq s \leq t \leq 1} \frac{\left|W(k)_{s, t}^{1} \otimes Z(k)_{s, t}^{1}\right|^{2 m}}{(t-s)^{1+4 m \alpha}} d s d t\right)^{2}\right] \\
& \leq \frac{1}{2} \iint_{0 \leq s \leq t \leq 1} \frac{\mathbb{E}\left[\left|W(k)_{s, t}^{1} \otimes Z(k)_{s, t}^{1}\right|^{4 m}\right]}{(t-s)^{2+8 m \alpha}} d s d t \\
& \leq C_{m, d} \iint_{0 \leq s \leq t \leq 1} \frac{\left(2^{-k}\right)^{2 m} \wedge(t-s)^{4 m}}{(t-s)^{2+8 m \alpha}} d s d t \\
& \leq C_{\alpha, m, d}\left(\frac{1}{2^{(4 m-8 m \alpha-1) / 2}}\right)^{k} .
\end{aligned}
$$

Here, we used (5.6) for the last inequality.

Applying Proposition 4.1 to $\left\|W(k)^{1} \otimes Z(k)\right\|_{2 \alpha, 2 m-B}^{2 m} \in \hat{\mathcal{C}}_{4 m}$, we obtain from (5.17) that

$$
\|\| W(k)^{1} \otimes Z(k)\left\|_{2 \alpha, 2 m-B}^{2 m}\right\|_{q, r} \leq C_{q, r, \alpha, m, d}\left(\frac{1}{2^{(4 m-8 m \alpha-1) / 4}}\right)^{k}
$$

for some positive constant $C_{q, r, \alpha, m, d}$ independent of $k$. Hence, if $4 m-8 m \alpha-1>0$, the right hand side of (5.14) is summable.

Finally, we consider (5.13). In this case we have

$$
\mathbb{E}\left[\left|J[z(k), w(k)]_{s, t}\right|^{2}\right] \leq C_{d}\left(\frac{1}{2^{k}} \wedge(t-s)^{2}\right), \quad(s, t) \in \triangle,
$$

for some positive constant $C_{d}$. The right hand side of (5.18) is the same as in (5.16). Hence, (5.18) implies summability of (5.13).

Now we give a sketch of the proof of (5.18). If $(j-1) / 2^{k} \leq s \leq t \leq j / 2^{k}$ for some $j$, then we can easily verify (5.18) by using (5.4). If $(j-1) / 2^{k} \leq s \leq j / 2^{k}$ and $(l-1) / 2^{k} \leq t \leq l / 2^{k}$ with $j<l$, then we can verify (5.18) by using Chen's identity as follows:

$$
\begin{gathered}
J[z(k), w(k)]_{s, t}=J[z(k), w(k)]_{s, j / 2^{k}}+\sum_{j+1 \leq i \leq l-1} J[z(k), w(k)]_{(i-1) / 2^{k}, i / 2^{k}} \\
+J[z(k), w(k)]_{(l-1) / 2^{k}, t}+Z(k)_{s, j / 2^{k}}^{1} \otimes W(k)_{j / 2^{k}, t}^{1} .
\end{gathered}
$$

From this we can see (5.18). Thus, we have shown the lemma.

As a corollary, we have quasi-continuity of Brownian rough path, which was first given by Aida [1] in a slightly stronger form.

Corollary 5.4. Let $\alpha$ and $m$ be as in Lemmas 5.2 and 5.3. Then, the mapping $w \mapsto W=S_{2}(w)$ is $\infty$-quasi continuous.

Proof. We will show $S_{2}: \mathcal{W} \rightarrow G \Omega_{\alpha, 4 m}^{B}\left(\mathbf{R}^{d}\right)$ is quasi-continuous. Let $n \in \mathbf{N}_{+}$be arbitrary. Then, we can find $N=N(n)$ such that

$$
\sum_{k=N}^{\infty} \mathrm{c}_{n, n}\left(\mathcal{A}_{\alpha, 4 m}^{k}\right)<\frac{1}{4 n}, \quad \max _{1 \leq i \leq 3} \sum_{k=N}^{\infty} \mathrm{c}_{n, n}\left(\mathcal{B}(i)_{2 \alpha, 2 m}^{k}\right)<\frac{1}{4 n} .
$$

On $\left(\bigcup_{k=N}^{\infty} \mathcal{A}_{\alpha, 4 m}^{k}\right)^{c}=\bigcap_{k=N}^{\infty}\left(\mathcal{A}_{\alpha, 4 m}^{k}\right)^{c},\left\{W^{1}(k)\right\}_{k}$ converges uniformly as $k \rightarrow$ $\infty$. For each $k, w \mapsto W^{1}(k)$ is clearly continuous. Hence, outside $\bigcup_{k=N}^{\infty} \mathcal{A}_{\alpha, 4 m}^{k}$, 
$W^{1}=S_{2}(w)^{1}$ is continuous in $w$. Similarly, outside $\bigcup_{i=1}^{3} \bigcup_{k=N}^{\infty} \mathcal{B}(i)_{\alpha, 4 m}^{k}, W^{2}=$ $S_{2}(w)^{2}$ is continuous in $w$.

Therefore, the set $\bigcup_{k=N}^{\infty} \mathcal{A}_{\alpha, 4 m}^{k} \cup\left[\bigcup_{i=1}^{3} \bigcup_{k=N}^{\infty} \mathcal{B}(i)_{\alpha, 4 m}^{k}\right]$ has $(n, n)$-capacity smaller than $1 / n$, due to subadditivity of the capacity, and its complement is a subset of $\mathcal{Z}_{\alpha, 4 m}$. Moreover, on its complement, $S_{2}$ is continuous. This proves the corollary.

Remark 5.5. If we assume (2.3) then all the assumptions for $\alpha, m$ in all the results in this section are satisfied. The condition (2.3) might not be the best possible, but is sufficient for our purpose. Because what is really needed is as follows: for any $\alpha \in(1 / 3,1 / 2)$, sufficiently large $m \in \mathbf{N}_{+}$satisfies the assumptions of these results.

\section{Quasi-sure analysis of SDE VIa ROUGh PATH}

We study SDE (2.1), which involves a small positive parameter $\varepsilon \in(0,1]$. In this section $\varepsilon$ is fixed, however. Hence, readers who like it simple may set $\varepsilon=1$.

Next we introduce a rough differential equation (RDE) associated with the vector fields $V_{i}$ 's and express $y^{\varepsilon}$ in terms of the Lyons-Itô map. For a while we assume $V_{i}$ 's are only $C_{b}^{3}$. Set $\lambda \in C_{0}^{1-H}([0,1], \mathbf{R})$ by $\lambda_{t}=t$. For $1 / 3<\alpha<1 / 2$, we denote by $\tau_{\lambda}: G \Omega_{\alpha}^{H}\left(\mathbf{R}^{d}\right) \rightarrow G \Omega_{\alpha}^{H}\left(\mathbf{R}^{d+1}\right)$ the Young pairing formally given by $X \mapsto(X, \lambda)$. We denote by $\Phi_{\varepsilon}: G \Omega_{\alpha}^{H}\left(\mathbf{R}^{d+1}\right) \rightarrow G \Omega_{\alpha}^{H}\left(\mathbf{R}^{n}\right)$ the Lyons-Itô map associated with the coefficients $\left[V_{1}, \ldots, V_{d}, V_{0}(\varepsilon, \cdot)\right]$ and the starting point $a$, which is continuous by Lyons' continuity theorem. If $X=S_{2}(x)$ for a 1-Hölder continuous path, then $a+\Phi_{\varepsilon}\left(\tau_{\lambda}(X)\right)^{1}$ solves the following ODE in Riemann-Stieltjes sense:

$$
d z_{t}=\sum_{i=1}^{d} V_{i}\left(z_{t}\right) d x_{t}^{i}+V_{0}\left(\varepsilon, z_{t}\right) d t, \quad z_{0}=a .
$$

Hence, by Wong-Zakai's approximation theorem, $y^{\varepsilon}=a+\Phi_{\varepsilon}\left(\tau_{\lambda}(\varepsilon W)\right)^{1}$ for a.a.w. Thus, the solution of the scaled SDE is obtained via rough path theory. Note also that this shows $y^{\varepsilon}$ is a $C^{\alpha-H}\left([0,1], \mathbf{R}^{n}\right)$-valued Wiener functional.

Proposition 6.1. Assume that $V_{1}, \ldots, V_{d}, V_{0}(\varepsilon, \cdot)$ are $C_{b}^{3}$ and let $\left(y_{t}^{\varepsilon}\right)_{0 \leq t \leq 1}$ be the solution of SDE (2.1). Then, for any $1 / 3<\alpha<1 / 2$, the mapping

$$
\mathcal{W} \ni w \mapsto y^{\varepsilon} \in C^{\alpha-H}\left([0,1], \mathbf{R}^{n}\right)
$$

admits an $\infty$-quasi-redefinition, which is explicitly given by $a+\Phi_{\varepsilon}\left(\tau_{\lambda}(\varepsilon W)\right)^{1}$.

Proof. For given $\alpha$, we can find $\left(\alpha^{\prime}, m\right)$ as in (2.3) such that $\alpha<\alpha^{\prime}$ and $\alpha^{\prime}-$ $(4 m)^{-1} \geq \alpha$. By Proposition [3.1, $G \Omega_{\alpha^{\prime}, 4 m}^{B}\left(\mathbf{R}^{d}\right)$ is continuously embedded in $G \Omega_{\alpha}^{H}\left(\mathbf{R}^{d}\right)$. To keep notation simple, we will not write this injection explicitly.

For all $w \in \mathcal{W}$ and $k \in \mathbf{N}_{+}$, we have $y(k)^{\varepsilon}=a+\Phi_{\varepsilon}\left(\tau_{\lambda}(\varepsilon W(k))\right)^{1}$, where $y(k)^{\varepsilon}$ is the unique solution of

$$
d y(k)_{t}^{\varepsilon}=\sum_{i=1}^{d} V_{i}\left(y(k)_{t}^{\varepsilon}\right) \varepsilon d w(k)_{t}+V_{0}\left(\varepsilon, y(k)_{t}^{\varepsilon}\right) d t, \quad y(k)_{0}^{\varepsilon}=a .
$$

Since we assume $C_{b}^{3}$, we may use Wong-Zakai's theorem. This theorem says $\sup _{0 \leq t \leq 1}\left|y(k)_{t}^{\varepsilon}-y_{t}^{\varepsilon}\right| \rightarrow 0$ almost surely as $k \rightarrow \infty$. On the other hand, by Lyons' continuity theorem, Proposition 3.1, and Corollary [5.4 $\Phi_{\varepsilon}\left(\tau_{\lambda}(\varepsilon W(k))\right)^{1}$ converges to $\Phi_{\varepsilon}\left(\tau_{\lambda}(\varepsilon W)\right)^{1}$ in $\alpha$-Hölder norm not just almost surely, but also quasi-surely. Hence, we have $y^{\varepsilon}=a+\Phi_{\varepsilon}\left(\tau_{\lambda}(\varepsilon W)\right)^{1}$ a.s. Again by Lyons' continuity theorem, 
Proposition 3.1 and Corollary [5.4, the right hand side is $\infty$-quasi-continuous in $w$. This implies that $a+\Phi_{\varepsilon}\left(\tau_{\lambda}(\varepsilon W)\right)^{1}$ is an $\infty$-quasi-redefinition of $y^{\varepsilon}$.

Remark 6.2. J. Ren [24] studied the quasi-sure refinement of Wong-Zakai's approximation theorem. The proof of the above theorem is a new proof of Ren's result. In fact, it is an improvement since we only assumed $C_{b}^{3}$, not $C_{b}^{\infty}$, for the coefficients.

Remark 6.3. The above proposition can be regarded as a new proof of MalliavinNualart's result in 22. Here, we give a quick remark about it. Assume that the coefficients $V_{i}$ 's are $C_{b}^{\infty}$. Then, for each $t, y_{t}^{\varepsilon} \in \mathbf{D}_{\infty}\left(\mathbf{R}^{n}\right)$ and therefore it admits an $\infty$-quasi-redefinition by a well-known general theory. J. Ren 24 proved that there exists a nice modification $\hat{y}^{\varepsilon}$ of $y^{\varepsilon}$ such that, for each $t, \hat{y}_{t}^{\varepsilon}$ is an $\infty$-quasi-redefinition of $y_{t}^{\varepsilon}$. (This process $\hat{y}^{\varepsilon}$ is the limit of Ren's refinement of Wong-Zakai's theorem in 24.)

But, it is more difficult to prove that the path space-valued functional $y^{\varepsilon}$ admits an $\infty$-quasi-redefinition. Malliavin and Nualart [22] proved that $w \mapsto y^{\varepsilon}$ admits an $\infty$-quasi-redefinition. (It might be possible to prove this by extending Ren's method, though it is not explicitly written in [24.) More precisely, they proved that Wiener functional

$$
\mathcal{W} \ni w \mapsto\left[[0,1] \times \mathbf{R}^{n} \ni(t, a) \mapsto y_{t}^{\varepsilon}=y^{\varepsilon}(t, a)\right],
$$

which takes values in a space of two-parameter functions, admits an $\infty$-quasiredefinition. But, this is not the point here.

Their method is as follows. Firstly, they introduced a UMD Banach space with certain nice properties and showed $y^{\varepsilon}$ takes values in it. Secondly, they extended Malliavin calculus so that it applies to Wiener functionals which take values in the UMD Banach space. Thirdly, they regarded SDE (2.1) as an equation that takes values in the UMD Banach space and took $\mathcal{H}$-derivative of it to prove that $y^{\varepsilon}$ is a UMD Banach space-valued $\mathbf{D}_{\infty}$-Wiener functional. Then, the same argument as in the case of real-valued $\mathbf{D}_{\infty}$-Wiener functionals applies to $y^{\varepsilon}$, too, and existence of $\infty$-redefinition is shown.

Note that, in their method, smoothness of the coefficients needs to be assumed. In our Proposition 6.1, however, we only assumed $C_{b}^{3}$, which is probably astonishing if we do not know rough path theory. In this sense, this is an improvement of Malliavin-Nualart's result.

Since Lyons-Itô map is continuous in the initial value $a$, too, it is clearly possible to extend Proposition 6.1 to the case of (6.1), by introducing certain Hölder-type norm on the two-parameter function space. It is not clear, however, whether we have really improved the results in [22] or not, mainly because we do not know how strong the UMD Banach norm is, compared to the Hölder-type norm. (Practically, such slight difference of norms is not important, however.)

Now, we discuss pinned diffusion measures. By using the arguments we developed so far, we can obtain pinned diffusion processes via rough path theory. This result was first proved in Inahama [15, but the argument here is much more straightforward, due to quasi-continuity of $W$ (Corollary 5.4).

From now on we assume $V_{i}(0 \leq i \leq d)$ are $C_{b}^{\infty}$. We assume further that the vector fields $\left\{V_{1}, \ldots, V_{d} ; V_{0}^{\varepsilon}\right\}$ satisfy hypoellipticity condition at any $a$. Here, $V_{0}^{\varepsilon}=V_{0}(\varepsilon, \cdot)$. Then, the solution $y_{t}^{\varepsilon}=y^{\varepsilon}(t, a)$ of SDE (2.1) has a density $p_{t}^{\varepsilon}\left(a, a^{\prime}\right)$ 
for all $t>0, \varepsilon>0$, and $a \in \mathbf{R}^{n}$, that is, for any Borel set $A \subset \mathbf{R}^{n}, \mathbb{P}\left(y^{\varepsilon}(t, a) \in A\right)=$ $\int_{A} p_{t}^{\varepsilon}\left(a, a^{\prime}\right) d a^{\prime}$. By Watanabe's theory, $p_{t}^{\varepsilon}\left(a, a^{\prime}\right)=\mathbb{E}\left[\delta_{a^{\prime}}\left(y^{\varepsilon}(t, a)\right)\right]$. (See Section 5.9 -5.10 , 14.) In this case $p_{1}^{\varepsilon}\left(a, a^{\prime}\right)>0$ for any $a, a^{\prime}$.

Under this condition, $\mathbb{Q}_{a, a^{\prime}}^{\varepsilon}$ exists as a probability measure on $C_{a, a^{\prime}}\left([0,1], \mathbf{R}^{n}\right):=$ $\left\{x \in C\left([0,1], \mathbf{R}^{n}\right) \mid x_{0}=a, x_{1}=a^{\prime}\right\}$ characterized by the following equation: for any $l \in \mathbf{N}_{+}$, any partition $\left\{0=t_{0}<t_{1}<\cdots<t_{l}<t_{l+1}=1\right\}$ of $[0,1]$, and any $f \in \mathcal{S}\left(\mathbf{R}^{n l}\right)$,

$$
\begin{aligned}
& \int f\left(x_{t_{1}}, \ldots, x_{t_{l}}\right) \mathbb{Q}_{a, a^{\prime}}^{\varepsilon}(d x) \\
& \quad=p_{1}^{\varepsilon}\left(a, a^{\prime}\right)^{-1} \int_{\left(\mathbf{R}^{n}\right)^{l}} f\left(a_{1}, \ldots, a_{l}\right) \prod_{j=1}^{l+1} p_{t_{j}-t_{j-1}}^{\varepsilon}\left(a_{j-1}, a_{j}\right) \prod_{j=1}^{l} d a_{j} .
\end{aligned}
$$

Here, $a_{0}=a$ and $a_{l+1}=a^{\prime}$ by convention. Actually, by Proposition 6.4 below, $\mathbb{Q}_{a, a^{\prime}}^{\varepsilon}$ turns out to be a probability measure on $C_{a, a^{\prime}}^{\alpha-H}\left([0,1], \mathbf{R}^{n}\right)$ for any $1 / 3<\alpha<1 / 2$.

Let $\theta_{a, a^{\prime}}^{\varepsilon}$ be a Borel finite measure on $\mathcal{W}$ that corresponds to $\delta_{a^{\prime}}\left(y^{\varepsilon}(1, a)\right)$ via Sugita's theorem (Proposition 4.5). We denote by $\hat{\theta}_{a, a^{\prime}}^{\varepsilon}$ its normalization. Let $\alpha, m$ be as in (2.3). We set $\mu_{a, a^{\prime}}^{\varepsilon}=\left(\varepsilon S_{2}\right)_{*} \theta_{a, a^{\prime}}^{\varepsilon}$ and $\hat{\mu}_{a, a^{\prime}}^{\varepsilon}=\left(\varepsilon S_{2}\right)_{*} \hat{\theta}_{a, a^{\prime}}^{\varepsilon}$. Since $\mathcal{Z}_{\alpha, 4 m}^{c}$ is slim, the lift map $\varepsilon S_{2}$ is well defined with respect to these measures. Thus, we obtained measures on $G \Omega_{\alpha, 4 m}^{B}\left(\mathbf{R}^{n}\right)$. It is easy to see that the support of $\hat{\mu}_{a, a^{\prime}}^{\varepsilon}$ is in fact contained in the closed subset $\left\{X \mid a+\Phi_{\varepsilon}\left(\tau_{\lambda}(X)\right)=a^{\prime}\right\}$.

The following proposition states that the pinned diffusion measure can be obtained as a continuous image of Lyons-Itô map. Note that everywhere hypoellipticity was assumed to guarantee existence of $\mathbb{Q}_{a, a^{\prime}}^{\varepsilon}$, but none of (H0)-(H2) was.

Proposition 6.4. Keep all the notation and assumptions as above. Then, the pushforward measure $\left(a+\Phi_{\varepsilon}^{1} \circ \tau_{\lambda}\right)_{*} \hat{\mu}_{a, a^{\prime}}^{\varepsilon}$ is the pinned diffusion measure $\mathbb{Q}_{a, a^{\prime}}^{\varepsilon}$. Here, $\Phi_{\varepsilon}^{1}$ denotes the first level of the Itô map $\Phi_{\varepsilon}$.

Proof. For simplicity, we write $\tilde{y}^{\varepsilon}=a+\Phi_{\varepsilon}\left(\tau_{\lambda}\left(\varepsilon S_{2}(w)\right)\right)^{1}$ as in the proof of Proposition 6.1. By Proposition 6.1 this is an $\infty$-quasi-redefinition of $y^{\varepsilon}$. It is sufficient to prove that $\left(a+\Phi_{\varepsilon}^{1} \circ \tau_{\lambda} \circ \varepsilon S_{2}\right)_{*} \theta_{a, a^{\prime}}^{\varepsilon}=p_{1}^{\varepsilon}\left(a, a^{\prime}\right) \cdot \mathbb{Q}_{a, a^{\prime}}^{\varepsilon}$. This fact is well known in quasi-sure analysis, but we give a proof here for the reader's convenience.

Let $\chi: \mathbf{R}^{n} \rightarrow \mathbf{R}$ be $C^{\infty}$ with compact support such that $\chi \geq 0$ and $\int_{\mathbf{R}^{n}} \chi(\xi) d \xi=$ 1. For $k \in \mathbf{N}_{+}$, set $\psi_{k}(\xi)=k^{n} \chi\left(k\left(\xi-a^{\prime}\right)\right)$. Then, for any function $g: \mathbf{R}^{n} \rightarrow$ $\mathbf{R}$ which is continuous around $a^{\prime}$, we have $\lim _{k \rightarrow \infty} \int_{\mathbf{R}^{n}} \psi_{k}(\xi) g(\xi) d \xi=g\left(a^{\prime}\right)$. In particular, $\lim _{k \rightarrow \infty} \psi_{k}=\delta_{a^{\prime}}$ in $\mathcal{S}^{\prime}\left(\mathbf{R}^{n}\right)$.

Take any partition $\left\{0=t_{0}<t_{1}<\cdots<t_{l}<t_{l+1}=1\right\}$ of $[0,1]$ and any $f \in \mathcal{S}\left(\mathbf{R}^{n l}\right)$. By Proposition 4.5, we have

$$
\begin{array}{rl}
\int_{\mathcal{W}} & f\left(\tilde{y}_{t_{1}}^{\varepsilon}, \ldots, \tilde{y}_{t_{l}}^{\varepsilon}\right) \theta_{a, a^{\prime}}^{\varepsilon}(d w) \\
& =\mathbb{E}\left[f\left(y_{t_{1}}^{\varepsilon}, \ldots, y_{t_{l}}^{\varepsilon}\right) \cdot \delta_{a^{\prime}}\left(y_{t}^{\varepsilon}\right)\right]=\lim _{k \rightarrow \infty} \mathbb{E}\left[f\left(y_{t_{1}}^{\varepsilon}, \ldots, y_{t_{l}}^{\varepsilon}\right) \psi_{k}\left(y_{t}^{\varepsilon}\right)\right] \\
& =\lim _{k \rightarrow \infty} \int_{\left(\mathbf{R}^{n}\right)^{l+1}} f\left(a_{1}, \ldots, a_{l}\right) \psi_{k}\left(a_{l+1}\right) \prod_{j=1}^{l+1} p_{t_{j}-t_{j-1}}^{\varepsilon}\left(a_{j-1}, a_{j}\right) \prod_{j=1}^{l+1} d a_{j} \\
& =\int_{\left(\mathbf{R}^{n}\right)^{l}} f\left(a_{1}, \ldots, a_{l}\right) \prod_{j=1}^{l} p_{t_{j}-t_{j-1}}^{\varepsilon}\left(a_{j-1}, a_{j}\right) p_{t_{l+1}-t_{l}}^{\varepsilon}\left(a_{l}, a^{\prime}\right) \prod_{j=1}^{l} d a_{j}
\end{array}
$$

where $a_{0}=a$. This completes the proof. 


\section{Proofs of large deviations}

The aim of this section is to prove our main results. The most important one is Theorem 2.1 (i), which will be shown in Subsections 7.1 and 7.2

The second assertion of Theorem 2.1 is immediate from the first one, because the weight of the whole set equals $p_{1}^{\varepsilon}\left(a, a^{\prime}\right)=\mathbb{E}\left[\delta_{a^{\prime}}\left(y^{\varepsilon}(1, a)\right)\right]$ and the whole set is both open and closed.

As an immediate consequence of Theorem 2.1 (i), we can show the following corollary. This can be considered as a special case of Theorem 2.1, 28. After normalization, we obtain Theorem 2.2 .

Corollary 7.1. Keep the same notation and assumptions as in Theorem 2.1 (i). Then, the pushforward measure of $\theta_{a, a^{\prime}}^{\varepsilon}$ by the map $\tilde{y}^{\varepsilon}$ satisfies a large deviation principle with a good rate function $J^{\prime}(y)$, where $J^{\prime}(y)=\inf \left\{\|h\|_{\mathcal{H}}^{2} / 2 \mid h \in\right.$ $\mathcal{K}^{a, a^{\prime}}$ with $\left.y=\phi^{0}(h)\right\}$ if $y=\phi^{0}(h)$ for some $h \in \mathcal{K}^{a, a^{\prime}}$ and $J^{\prime}(y)=\infty$ if no such $h \in \mathcal{K}^{a, a^{\prime}}$ exists.

Proof of Corollary 7.1 and Theorem 2.2 . There exists $\alpha^{\prime} \in(\alpha, 1 / 2)$ and $m \in \mathbf{N}_{+}$ such that $\alpha^{\prime}-(4 m)^{-1} \geq \alpha$ and (2.3) hold. Then, we can use Theorem 2.1 above and we have a continuous embedding $G \Omega_{\alpha^{\prime}, 4 m}^{B}\left(\mathbf{R}^{d}\right) \hookrightarrow G \Omega_{\alpha}^{H}\left(\mathbf{R}^{d}\right)$ by Proposition 3.1. Of course, the Young pairing $\tau_{\lambda}: G \Omega_{\alpha}^{H}\left(\mathbf{R}^{d}\right) \rightarrow G \Omega_{\alpha}^{H}\left(\mathbf{R}^{d+1}\right)$ is continuous.

Let us give a remark on continuity of the Lyons-Itô map $\Phi_{\varepsilon}$ since it depends on $\varepsilon$, too. The coefficient $V_{0}$ for the drift term depends on $\varepsilon$ and $C_{b}^{\infty}\left([0,1] \times \mathbf{R}^{n}\right)$. Hence, by Taylor expansion, we have

$$
\sup _{\xi:|\xi| \leq R}\left|\nabla_{(\xi)}^{i} V_{0}(\varepsilon, \xi)-\nabla_{(\xi)}^{i} V_{0}\left(\varepsilon_{0}, \xi\right)\right| \rightarrow 0 \quad \text { as } \varepsilon \rightarrow \varepsilon_{0}
$$

for any $R>0$ and $0 \leq i \leq 3$. Here, $\nabla_{(\xi)}$ stands for the gradient in $\xi$-variable.

Under this kind of uniform convergence of the $\varepsilon$-dependent coefficient, a slight generalization of Lyons' continuity theorem holds, which claims that the mapping

$$
[0,1] \times G \Omega_{\alpha}^{H}\left(\mathbf{R}^{d+1}\right) \ni(\varepsilon, Z) \mapsto \Phi_{\varepsilon}(Z) \in G \Omega_{\alpha}^{H}\left(\mathbf{R}^{n}\right)
$$

is continuous. It immediately follows that the mapping

$$
[0,1] \times G \Omega_{\alpha^{\prime}, 4 m}^{B}\left(\mathbf{R}^{d}\right) \ni(\varepsilon, X) \mapsto a+\Phi_{\varepsilon}\left(\tau_{\lambda}(X)\right)^{1} \in C_{a}^{\alpha-H}\left([0,1], \mathbf{R}^{d}\right)
$$

is continuous, too. Now we can use Proposition 6.4 and a slight generalization of the contraction principle for large deviations (see Lemma 3.9, [16] for example). This completes the proof.

In the same way, the proof of Theorem 2.2 follows from Proposition 6.4 and Theorem 2.1 (ii).

Before we prove Theorem 2.1(i), let us recall that a Schilder-type large deviation for the laws of scaled Brownian rough path $\varepsilon W$ holds on $G \Omega_{\alpha^{\prime}}^{H}\left(\mathbf{R}^{d}\right)$ as $\varepsilon \searrow 0$ for any $1 / 3<\alpha^{\prime}<1 / 2$ with a good rate function $I_{S c h}$ (see Friz-Victoir [8]). Here, $I_{S c h}(X)=\|h\|_{\mathcal{H}}^{2} / 2$ if $X=S_{2}(h)$ for some $h \in \mathcal{H}$ and $I_{S c h}(X)=\infty$ if otherwise. For $\alpha<\alpha^{\prime}<1 / 2$, there is a continuous embedding $G \Omega_{\alpha^{\prime}}^{H}\left(\mathbf{R}^{d}\right) \hookrightarrow G \Omega_{\alpha, 4 m}^{B}\left(\mathbf{R}^{d}\right)$. Hence, by the contraction principle, the Schilder-type large deviation also holds on $G \Omega_{\alpha, 4 m}^{B}\left(\mathbf{R}^{d}\right)$ and $I_{S c h}$ is still good on $G \Omega_{\alpha, 4 m}^{B}\left(\mathbf{R}^{d}\right)$. We can easily verify from this that $I$ defined in Section 2 is lower semicontinuous and good (i.e., the level set $\{X \mid I(X) \leq c\}$ is compact for all $0 \leq c<\infty)$. So is $\hat{I}$. 
7.1. Proof of Theorem 2.1 (i): Lower estimate. The aim of this subsection is to prove the lower estimate in Theorem 2.1 (i).

Before we start our proof, let us rewrite SDE (2.1) in matrix form. Set $\sigma: \mathbf{R}^{n} \rightarrow$ $\operatorname{Mat}(n, d)$ by $\left[V_{1}, \ldots, V_{d}\right]$, that is, the $j$ th column vector of $\sigma$ is $V_{j}$. We also write $V_{0}(\varepsilon, \xi)=b(\varepsilon, \xi)$. Then, SDE (2.1) can be rewritten as

$$
d y_{t}^{\varepsilon}=\sigma\left(y_{t}^{\varepsilon}\right) \circ \varepsilon d w_{t}+b\left(\varepsilon, y_{t}^{\varepsilon}\right) d t, \quad y_{0}^{\varepsilon}=a .
$$

This matrix form (7.1) is sometimes more convenient than (2.1). In the same way, ODE (2.2) is equivalent to the following ODE:

$$
d \phi_{t}^{0}=\sigma\left(\phi_{t}^{0}\right) d h_{t}+b\left(0, \phi_{t}^{0}\right) d t, \quad \phi_{0}^{0}=a .
$$

Let $\left(y_{t}^{\varepsilon}(w)\right)_{0 \leq t \leq 1}$ be the solution of $\operatorname{SDE}(7.1)$. For $h \in \mathcal{H}$, we consider the Cameron-Martin shift of $y^{\varepsilon}$,

$$
d y_{t}^{\varepsilon, h}=\sigma\left(y_{t}^{\varepsilon, h}\right)\left[\circ \varepsilon d w_{t}+d h_{t}\right]+b\left(\varepsilon, y_{t}^{\varepsilon, h}\right) d t, \quad y_{0}^{\varepsilon, h}=a .
$$

An $\infty$-quasi-continuous version of $y^{\varepsilon, h}$ is given by $a+\Phi_{\varepsilon}\left(\tau_{\lambda}\left(S_{2}(\varepsilon w+h)\right)\right)$ by Lemma 5.1 and Proposition 6.1.

Non-degeneracy of the Malliavin covariance matrix of the solution of SDE under ellipticity condition is in Section 6.1.5, Shigekawa 25, for example. By repeating the same proof, carefully taking $\varepsilon$-dependency into account, we can verify that $\left(y_{1}^{\varepsilon, h}-a^{\prime}\right) / \varepsilon$ is uniformly non-degenerate in the sense of Malliavin as $\varepsilon \searrow 0$. (This fact was already shown in [29] when the drift term is of the form $b(\varepsilon, \xi)=\varepsilon^{2} \hat{b}(\xi)$ for an $\varepsilon$-independent vector field $\hat{b}$. The definition of uniform non-degeneracy is in p. 10, 29. When we try to extend our method to the hypoelliptic case, this part becomes very difficult.)

Moreover, in the same way as in Watanabe [29, we have the following asymptotics for $y_{1}^{\varepsilon, h}$ as $\varepsilon \searrow 0$ :

$$
y_{1}^{\varepsilon, h}=\phi^{0}(h)_{1}+\varepsilon \phi^{1}(\cdot ; h)_{1}+O\left(\varepsilon^{2}\right) \quad \text { in } \mathbf{D}_{\infty}\left(\mathbf{R}^{n}\right) .
$$

Watanabe obtained the expansion up to any order when the drift term is of the form $b(\varepsilon, \xi)=\varepsilon^{2} \hat{b}(\xi)$. But we only need an expansion up to second order. Modification for the case of general $b(\varepsilon, \xi)$ is easy. Here, $\phi_{t}^{1}=\phi^{1}(w ; h)_{t}$ is the unique solution of the following equation:

$$
d \phi_{t}^{1}-\nabla \sigma\left(\phi_{t}^{0}\right)\left\langle\phi_{t}^{1}, d h_{t}\right\rangle-\nabla_{(\xi)} b\left(0, \phi_{t}^{0}\right)\left\langle\phi_{t}^{1}\right\rangle d t=\sigma\left(\phi_{t}^{0}\right) d w_{t}+\partial_{\varepsilon} b\left(0, \phi_{t}^{0}\right) d t, \quad \phi_{0}^{1}=0 .
$$

Here, $\nabla_{(\xi)}$ denotes the (partial) gradient with respect to the second variable. $\partial_{\varepsilon}$ should be understood in a similar way.

Now we give an explicit expression for $\phi_{t}^{1}$. Let $M_{t}=M(h)_{t}$ be a unique solution of the following $n \times n$ matrix-valued ODE:

$$
d M_{t}=\left[\nabla \sigma\left(\phi_{t}^{0}\right)\left\langle\bullet, d h_{t}\right\rangle+\nabla_{(\xi)} b\left(0, \phi_{t}^{0}\right)\langle\bullet\rangle d t\right] \cdot M_{t}, \quad M_{0}=\operatorname{Id}_{n} .
$$

Then, $M_{t}$ is invertible for all $t$ and we have

$$
\phi_{t}^{1}=M_{t} \int_{0}^{t} M_{s}^{-1}\left[\sigma\left(\phi_{s}^{0}\right) d w_{s}+\partial_{\varepsilon} b\left(0, \phi_{s}^{0}\right) d s\right] .
$$

Hence, as a functional in $w, \phi_{1}^{1}$ is an $\mathbf{R}^{n}$-valued Gaussian random variable with mean $M_{1} \int_{0}^{1} M_{s}^{-1} \nabla_{\varepsilon} b\left(0, \phi_{s}^{0}\right) d s$ and covariance $M_{1} \int_{0}^{1} M_{s}^{-1} \sigma\left(\phi_{s}^{0}\right) \sigma\left(\phi_{s}^{0}\right)^{T}\left(M_{s}^{-1}\right)^{T} d s M_{1}^{T}$. Since we assumed ellipticity at the initial point $a, \sigma \sigma^{T}$ is positive and bounded away from 0 near $a$. Therefore, this covariance matrix is non-degenerate for any $h$. In particular, $\mathbb{E}\left[\delta_{0}\left(\phi_{1}^{1}\right)\right]>0$. 
Let $h \in \mathcal{K}^{a, a^{\prime}}$. By (a special case of) Theorem 2.3, [29, or Theorem 9.4, 14], and the uniform non-degeneracy, we have

$$
\lim _{\varepsilon \searrow 0} \delta_{0}\left(\frac{y_{1}^{\varepsilon, h}-a^{\prime}}{\varepsilon}\right)=\delta_{0}\left(\phi_{1}^{1}\right) \quad \text { in } \mathbf{D}_{q,-r}\left(\mathbf{R}^{n}\right)
$$

for some $q \in(1, \infty)$ and some $r \in \mathbf{N}$.

Lemma 7.2. $\mathcal{K}^{a, a^{\prime}} \cap \mathcal{W}^{*}$ is dense in $\mathcal{K}^{a, a^{\prime}}$. In other words, for any $h \in \mathcal{K}^{a, a^{\prime}}$, there exist $h_{j} \in \mathcal{K}^{a, a^{\prime}}\left(j \in \mathbf{N}_{+}\right)$such that $\left\langle h_{j}, \cdot\right\rangle_{\mathcal{H}} \in \mathcal{W}^{*}$ for all $j$ and $\lim _{j \rightarrow \infty}\left\|h_{j}-h\right\|_{\mathcal{H}}=$ 0 .

Proof. Set $F(h)=\phi^{0}(h)_{1}$. Then, $F: \mathcal{H} \rightarrow \mathbf{R}^{n}$ is Fréchet- $C^{1}$ and its Fréchet derivative $D F$ is given by

$$
D_{k} F(h)=\langle D F(h), k\rangle_{\mathcal{H}}=M_{1} \int_{0}^{1} M_{s}^{-1} \sigma\left(\phi^{0}(h)_{s}\right) k_{s}^{\prime} d s .
$$

Surjectivity of the linear map $D F(h): \mathcal{H} \rightarrow \mathbf{R}^{n}$ is equivalent to non-degeneracy of the "deterministic Malliavin covariance" matrix

$$
\left(\left\langle D F^{i}(h), D F^{j}(h)\right\rangle_{\mathcal{H}}\right)_{1 \leq i, j \leq n}=M_{1} \int_{0}^{1} M_{s}^{-1} \sigma\left(\phi^{0}(h)_{s}\right) \sigma\left(\phi^{0}(h)_{s}\right)^{T}\left(M_{s}^{-1}\right)^{T} d s M_{1}^{T},
$$

which has already been shown. Hence, we can use Lemma 7.3 below with $\mathcal{K}=\mathcal{H}$ and $\mathcal{L}=\mathcal{W}^{*}$.

Lemma 7.3. Let $\mathcal{K}$ be a real Hilbert space and $\xi \in \mathcal{K}$. Assume that (i) $F$ is an $\mathbf{R}^{n}$-valued Fréchet- $C^{1}$ map defined on a neighborhood of $\xi$ with a bounded derivative $D F$ and (ii) $D F(\xi): \mathcal{K} \rightarrow \mathbf{R}^{n}$ is a surjective linear map. Let $\mathcal{L}$ be a real Banach space which is continuously and densely embedded in $\mathcal{K}$. Then, there exists $\xi_{j} \in$ $\mathcal{L}(j=1,2, \ldots)$ such that $\lim _{j \rightarrow \infty}\left\|\xi_{j}-\xi\right\|_{\mathcal{K}}=0$ and $F\left(\xi_{j}\right)=F(\xi)$ for all $j$.

Proof. Without loss of generality, we may assume $F(\xi)=0$. It is sufficient to show that, for sufficiently small $r>0$, there exists $\eta \in \mathcal{L} \cap B_{\mathcal{K}}(\xi, r)$ such that $F(\eta)=0$, where $B_{\mathcal{K}}(\xi, r)$ is the open $\mathcal{K}$-ball of radius $r$, centered at $\xi$. There exists an $n$ dimensional subspace $V \subset \mathcal{K}$ such that $\left.\nabla F(\xi)\right|_{V}: V \rightarrow \mathbf{R}^{n}$ is a linear bijection. Hence, we may apply inverse function theorem at $\xi$ to $\left.F\right|_{\xi+V}$, which is the restriction of $F$ onto the affine subspace $\xi+V$.

First, we consider the case for $n=1$. For any sufficiently small $r>0$, there exist $\chi_{0}, \chi_{1} \in(\xi+V) \cap B_{\mathcal{K}}(\xi, r)$ such that $F\left(\chi_{0}\right)>0$ and $F\left(\chi_{1}\right)<0$. Since $\mathcal{L}$ is dense in $\mathcal{K}$, we can find $\eta_{0}, \eta_{1} \in \mathcal{L} \cap B_{\mathcal{K}}(\xi, r)$ such that $F\left(\eta_{0}\right)>0$ and $F\left(\eta_{1}\right)<0$. Then, the line segment $\overline{\eta_{0} \eta_{1}}=\left\{\tau \eta_{0}+(1-\tau) \eta_{1} \mid 0 \leq \tau \leq 1\right\}$ is still in $\mathcal{L} \cap B_{\mathcal{K}}(\xi, r)$. Then, by the intermediate value theorem, there exists $\tau^{\prime} \in(0,1)$ such that $F\left(\tau^{\prime} \eta_{0}+\left(1-\tau^{\prime}\right) \eta_{1}\right)=0$. Thus, we have shown the case for $n=1$.

Next, we consider the case for $n=2$. For $0<r<r^{\prime}$, set $B_{\mathbf{R}^{n}}(0, r)=\{a \in$ $\left.\mathbf{R}^{n}|| a \mid<r\right\}, S_{r}^{1}=\left\{a \in \mathbf{R}^{n}|| a \mid=r\right\}$, and $A_{r, r^{\prime}}=\left\{a \in \mathbf{R}^{n}|r<| a \mid<r^{\prime}\right\}$. For sufficiently small $r>0, F\left((\xi+V) \cap B_{\mathcal{K}}(\xi, r)\right)$ is an open neighborhood of 0 and $f:=\left.F\right|_{(\xi+V) \cap B_{\mathcal{K}}(\xi, r)}$ is a diffeomorphism. There exists $\rho>0$ such that $B_{\mathbf{R}^{n}}(0,3 \rho) \subset F\left((\xi+V) \cap B_{\mathcal{K}}(\xi, r)\right)$. Then, there exist $N \in \mathbf{N}_{+}$and $\xi_{0}, \ldots, \xi_{N} \in S_{\rho}^{1}$ such that the following conditions are satisfied:

(1) $\xi_{i}$ and $\xi_{i+1}$ are adjacent in counter-clockwise order (with $\xi_{N+1}=\xi_{0}$ ), 
(2) the arc between $\xi_{i}$ and $\xi_{i+1}$ can be continuously deformed inside $A_{\rho / 2,2 \rho}$, with the end points being fixed, so that the image of the arc by $f^{-1}$ is deformed to the line segment $\overline{f^{-1}\left(\xi_{i}\right) f^{-1}\left(\xi_{i+1}\right)}$.

Then, there exists $\eta_{0}, \ldots, \eta_{N} \in \mathcal{L} \cap B_{\mathcal{K}}(\xi, r)$ such that the following conditions are satisfied:

(1) there exists a continuous deformation of $\bigcup_{i=0}^{N} \overline{f^{-1}\left(\xi_{i}\right) f^{-1}\left(\xi_{i+1}\right)}$ inside $B_{\mathcal{K}}(\xi, r)$, which deforms each $\overline{f^{-1}\left(\xi_{i}\right) f^{-1}\left(\xi_{i+1}\right)}$ to $\overline{\eta_{i} \eta_{i+1}}$.

(2) the image of the above continuous deformation by $F$ stays inside $A_{\rho / 3,3 \rho}$.

Obviously, $\bigcup_{i=0}^{N} \overline{\eta_{i} \eta_{i+1}}$ is a subset of the following (possibly degenerate) simplex

$$
\triangle:=\left\{\eta_{0}+\sum_{i=1}^{N} \tau_{i}\left(\eta_{i}-\eta_{0}\right) \mid \tau_{i} \geq 0 \text { for all } i \text { and } \sum_{i=1}^{N} \tau_{i} \leq 1\right\} .
$$

This is the convex hull of $\eta_{0}, \ldots, \eta_{N}$. Hence, $\triangle \subset B_{\mathcal{K}}(\xi, r) \cap \mathcal{L}$. It is easy to see that $\triangle$ admits a continuous deformation to a single point set $\left\{\eta_{0}\right\}$ without getting out of $\triangle$.

Combining them all, we have shown that $S_{\rho}^{1}$ is continuously deformed in $\mathbf{R}^{2}$ to a single point set $\left\{F\left(\eta_{0}\right)\right\}$. More precisely, there exists a continuous map $\phi$ : $[0,1] \times S_{\rho}^{1} \rightarrow \mathbf{R}^{2}$ such that $\phi(0, \cdot)=\operatorname{Id}_{S_{\rho}^{1}}$ and $\phi(1, \cdot)$ is constant. By Lemma 7.4 below, there exists $(s, a) \in[0,1] \times S_{\rho}^{1}$ such that $\phi(s, a)=0$. By way of construction of the deformation map, there must be $\eta \in \triangle$ such that $F(\eta)=\phi(s, a)=0$. This proves the case for $n=2$. The higher-dimensional cases can be shown in the same way.

The following topological lemma can be regarded as a higher-dimensional version of the intermediate value theorem.

Lemma 7.4. Let $B^{n}=\left\{a \in \mathbf{R}^{n}|| a \mid \leq 1\right\}$ be the $n$-dimensional closed ball and let $S^{n-1}=\left\{a \in \mathbf{R}^{n}|| a \mid=1\right\}$ be the $(n-1)$-dimensional sphere $(n \geq 2)$.

(i) Let $f:[0,1] \times S^{n-1} \rightarrow \mathbf{R}^{n}$ be a continuous map such that $f(0, \cdot)=\operatorname{Id}_{S^{n-1}}$ and $f(1, \cdot)$ is a constant map. Then, there exists $(s, a) \in[0,1] \times S^{k-1}$ such that $f(s, a)=0$.

(ii) Let $g: B^{n} \rightarrow \mathbf{R}^{n}$ be a continuous map such that $\left.g\right|_{S^{n-1}}=\operatorname{Id}_{S^{n-1}}$. Then, there exists $a \in B^{n}$ such that $g(a)=0$.

Proof. Since $f(1, \cdot)$ is constant, there exists a continuous map $g: B^{n} \rightarrow \mathbf{R}^{n}$ such that $\left.g\right|_{S^{n-1}}=\operatorname{Id}_{S^{n-1}}$ and $\operatorname{Im} f=\operatorname{Im} g$. Hence, it is sufficient to prove the second assertion. Assume that $g^{-1}(0)=\emptyset$. Then $g$ is continuous from $B^{n}$ to $\mathbf{R}^{n} \backslash\{0\}$ and we have the following commutative diagram:

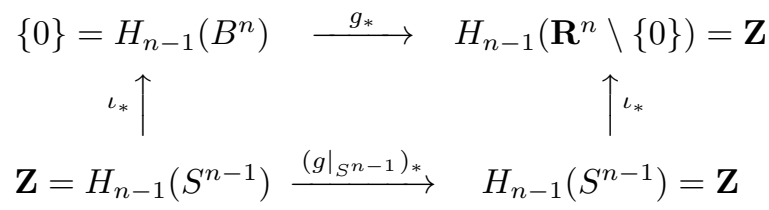

where $\iota$ stands for the injections. $g_{*} \circ\left(\iota: S^{n-1} \hookrightarrow B^{n}\right)_{*}$ is the zero map. On the other hand, $\left(\iota: S^{n-1} \hookrightarrow \mathbf{R}^{n} \backslash\{0\}\right)_{*} \circ\left(\left.g\right|_{S^{n-1}}\right)_{*}=\left(\iota: S^{n-1} \hookrightarrow \mathbf{R}^{n} \backslash\{0\}\right)_{*} \circ$ $\mathrm{id}_{H_{n-1}\left(S^{n-1}\right)}$ is an isomorphism, due to homotopy equivalence. This is a contradiction. Therefore, $g^{-1}(0) \neq \emptyset$. 
The lower estimate in Theorem 2.1 (i) is almost immediate from Lemma 7.2 above and the following proposition.

Proposition 7.5. Let $A \subset G \Omega_{\alpha, 4 m}^{B}\left(\mathbf{R}^{d}\right)$ be open and suppose that $S_{2}(h) \in A$ and $\langle h, \cdot\rangle_{\mathcal{H}} \in \mathcal{W}^{*} \cap \mathcal{K}^{a, a^{\prime}}$. Then, we have $-\|h\|_{\mathcal{H}}^{2} / 2 \leq \liminf \operatorname{lid}_{\varepsilon} \varepsilon^{2} \log \mu_{a, a^{\prime}}^{\varepsilon}(A)$.

Proof. For $R>0$, we set $B_{R}=\left\{X \in G \Omega_{\alpha, 4 m}^{B}\left(\mathbf{R}^{d}\right) \mid\left\|X^{i}\right\|_{i \alpha, 4 m / i-B}<R^{i} \quad(i=\right.$ $1,2)\}$ and $\hat{B}_{R}(H)=T_{h}\left(B_{R}\right)$, where $T_{h}$ is the Young translation by $h$ on $G \Omega_{\alpha, 4 m}^{B}\left(\mathbf{R}^{d}\right)$. Then, $\left\{\hat{B}_{R}(H) \mid R>0\right\}$ forms a fundamental system of open neighborhood around $H=S_{2}(h)$. Since $A$ is open, there exists $R_{0}>0$ such that $\hat{B}_{R_{0}}(H) \subset A$. We will estimate the weight of $\hat{B}_{R_{0}}(H)$ instead of that of $A$.

By Cameron-Martin formula, it holds that, for any $F \in \mathbf{D}_{\infty}$,

$$
\begin{aligned}
\mathbb{E}\left[F \delta_{a^{\prime}}\left(y_{1}^{\varepsilon}\right)\right] & =\mathbb{E}\left[\exp \left(-\frac{\langle h, w\rangle}{\varepsilon}-\frac{\|h\|_{\mathcal{H}}^{2}}{2 \varepsilon^{2}}\right) F\left(w+\frac{h}{\varepsilon}\right) \delta_{a^{\prime}}\left(y_{1}^{\varepsilon, h}\right)\right] \\
& =e^{-\|h\|_{\mathcal{H}}^{2} / 2 \varepsilon^{2}} \varepsilon^{-n} \mathbb{E}\left[e^{-\langle h, w\rangle / \varepsilon} F\left(w+\frac{h}{\varepsilon}\right) \delta_{0}\left(\frac{y_{1}^{\varepsilon, h}-a^{\prime}}{\varepsilon}\right)\right] .
\end{aligned}
$$

Here, we used the fact that $\delta_{0}(\varepsilon \xi)=\varepsilon^{-n} \delta_{0}(\xi)$.

We denote by $\nu^{\varepsilon}=\nu_{a, a^{\prime}}^{\varepsilon}$ the Borel measure corresponding to $\delta_{0}\left(\left(y_{1}^{\varepsilon, h}-a^{\prime}\right) / \varepsilon\right)$ via Sugita's theorem (Proposition 4.5). Then, we have

$$
\begin{aligned}
\mu_{a, a^{\prime}}^{\varepsilon}\left(\hat{B}_{R}(H)\right) & =\int_{\mathcal{W}} I_{\hat{B}_{R}(H)}(\varepsilon W) \theta_{a, a^{\prime}}^{\varepsilon}(d w) \\
& =e^{-\|h\|_{\mathcal{H}}^{2} / 2 \varepsilon^{2}} \varepsilon^{-n} \int_{\mathcal{W}} e^{-\langle h, w\rangle / \varepsilon} I_{B_{R / \varepsilon}}(W) \nu^{\varepsilon}(d w)
\end{aligned}
$$

for any $0<R<R_{0}$. Noting that $|\langle h, w\rangle / \varepsilon| \leq\|h\|_{\mathcal{W}^{*}}\|w / \varepsilon\|_{\mathcal{W}} \leq C\|h\|_{\mathcal{W}^{*}} R / \varepsilon^{2}$, where $C:=\sup _{w \neq 0}\|w\|_{\infty} /\|w\|_{\alpha, 4 m-B}$, we see that

$$
\begin{aligned}
\mu_{a, a^{\prime}}^{\varepsilon}\left(\hat{B}_{R}(H)\right) \geq & e^{-\|h\|_{\mathcal{H}}^{2} /\left(2 \varepsilon^{2}\right)} \varepsilon^{-n} e^{-C\|h\|_{\mathcal{W}^{*} R} / \varepsilon^{2}} \\
& \times \nu^{\varepsilon}\left(\left\{\left\|W^{i}\right\|_{i \alpha, 4 m / i-B}^{1 / i}<R / \varepsilon(i=1,2)\right\}\right) .
\end{aligned}
$$

Hence, it is sufficient to show that, for each fixed $R$,

$$
\lim _{\varepsilon \searrow 0} \nu^{\varepsilon}\left(\left\{\left\|W^{i}\right\|_{i \alpha, 4 m / i-B}^{1 / i}<R / \varepsilon(i=1,2)\right\}\right)=\nu^{\infty}(\mathcal{W})=\mathbb{E}\left[\delta_{0}\left(\phi_{1}^{1}\right)\right]>0,
$$

where $\nu^{\infty}$ stands for the Borel measure corresponding to $\delta_{0}\left(\phi_{1}^{1}\right)$ via Sugita's theorem. Indeed, (7.3) implies that

$$
\liminf _{\varepsilon \searrow 0} \varepsilon^{2} \log \mu_{a, a^{\prime}}^{\varepsilon}\left(\hat{B}_{R_{0}}(H)\right) \geq \liminf _{\varepsilon \searrow 0} \varepsilon^{2} \log \mu_{a, a^{\prime}}^{\varepsilon}\left(\hat{B}_{R}(H)\right) \geq-\frac{\|h\|_{\mathcal{H}}^{2}}{2}-C\|h\|_{\mathcal{W}^{*}} R .
$$

Letting $R \searrow 0$, we have $\liminf \operatorname{s\searrow 0}_{\varepsilon} \varepsilon^{2} \log \mu_{a, a^{\prime}}^{\varepsilon}\left(\hat{B}_{R_{0}}(H)\right) \geq-\|h\|_{\mathcal{H}}^{2} / 2$.

It remains to prove (7.3). Since $\delta_{0}\left(\left(y_{1}^{\varepsilon, h}-a^{\prime}\right) / \varepsilon\right) \rightarrow \delta_{0}\left(\phi_{1}^{1}\right)$ in $\mathbf{D}_{q^{\prime},-r}$ for some $q^{\prime} \in$ $(1, \infty)$ and $r \geq 0$, we have $\nu^{\varepsilon}(\mathcal{W})-\nu^{\infty}(\mathcal{W})=\mathbb{E}\left[1 \cdot\left\{\delta_{0}\left(\left(y_{1}^{\varepsilon, h}-a^{\prime}\right) / \varepsilon\right)-\delta_{0}\left(\phi_{1}^{1}\right)\right\}\right] \rightarrow 0$ as $\varepsilon \searrow 0$. Now, it is sufficient to show that

$$
\begin{aligned}
& \nu^{\varepsilon}\left(\left\{\left\|W^{i}\right\|_{i \alpha, 4 m / i-B}^{1 / i}<R / \varepsilon(i=1,2)\right\}^{c}\right) \leq \sum_{i=1,2} \nu^{\varepsilon}\left(\left\{\left\|W^{i}\right\|_{i \alpha, 4 m / i-B}^{1 / i} \geq R / \varepsilon\right\}\right) \\
& \leq \sum_{i=1,2}\left\|\delta_{0}\left(\left(y_{1}^{\varepsilon, h}-a^{\prime}\right) / \varepsilon\right)\right\|_{q^{\prime},-r} \cdot \mathrm{c}_{q, r}\left(\left\{\left\|W^{i}\right\|_{i \alpha, 4 m / i-B}^{1 / i} \geq R / \varepsilon\right\}\right) \rightarrow 0
\end{aligned}
$$


as $\varepsilon \searrow 0$. Here, $1 / q+1 / q^{\prime}=1$. Since $\left\|\delta_{0}\left(\left(y_{1}^{\varepsilon, h}-a^{\prime}\right) / \varepsilon\right)\right\|_{q^{\prime},-r}$ is bounded in $\varepsilon$, the problem is reduced to showing

$$
\lim _{\varepsilon \searrow 0} \mathrm{c}_{q, r}\left(\left\{\left\|W^{i}\right\|_{i \alpha, 4 m / i-B}^{1 / i} \geq R / \varepsilon\right\}\right)=0 \quad(i=1,2) .
$$

This will be shown in the next lemma below.

On an abstract Wiener space, weight of the complement set of the large ball admits Gaussian decay. This is called a large deviation estimate. A similar fact holds in rough path settings, too (for instance, see [7]). In this paper, however, we need a large deviation estimate for capacities, not for measures.

Lemma 7.6. Let $\alpha$ and $m$ be as in Theorem 2.1. For any $q \in(1, \infty)$ and $r \in \mathbf{N}$, there exist $c>0$ and $R_{1}>0$ such that

$$
\mathrm{c}_{q, r}\left(\left\{w \in \mathcal{W} \mid\left\|W^{i}\right\|_{i \alpha, 4 m / i-B}^{1 / i} \geq R\right\}\right) \leq e^{-c R^{2}} \quad \text { for any } R \geq R_{1} \text { and } i=1,2 .
$$

The constants $c$ and $R_{1}$ may depend on $\alpha, m, q, r$, but not on $R$.

Proof. First, let us check that there exists $c_{1}>0$ such that

$$
\mathbb{P}\left(\left\{w \in \mathcal{W} \mid\left\|W^{i}\right\|_{i \alpha, 4 m / i-B}^{1 / i} \geq R\right\}\right) \leq e^{-c_{1} R^{2}}
$$

holds for sufficiently large $R>0$. On the Hölder geometric rough path space $G \Omega_{\alpha}^{H}\left(\mathbf{R}^{d}\right)$, with any $1 / 3<\alpha<1 / 2$, this type of Gaussian decay is well known (see Friz and Oberhauser [7]). Next, take $\alpha^{\prime} \in(\alpha, 1 / 2)$. Then, due to the continuous embedding $G \Omega_{\alpha^{\prime}}^{H}\left(\mathbf{R}^{d}\right) \hookrightarrow G \Omega_{\alpha, 4 m}^{B}\left(\mathbf{R}^{d}\right)$, we can easily see (7.4) holds on the Besov geometric rough path space, too.

Take $\chi \in C_{b}^{\infty}(\mathbf{R} \rightarrow \mathbf{R})$ such that $\chi=0$ on $(-\infty, 0], \chi=1$ on $[1, \infty)$, and $\chi$ is non-decreasing. Set $G(w)=\left\|W^{1}\right\|_{\alpha, 4 m-B}^{4 m}$. Then, $G \in \mathbf{D}_{\infty}$ and $\chi\left(G-R^{4 m}+1\right) \in$ $\mathcal{F}_{q, r}\left(\left\{\left\|W^{1}\right\|_{\alpha, 4 m-B} \geq R\right\}\right)$ for any $q, r$ in the sense of (4.7), since $w \mapsto W$ is $\infty$ quasi-continuous by Corollary [5.4, By (4.8), we have

$$
\mathrm{c}_{q, r}\left(\left\{\left\|W^{1}\right\|_{\alpha, 4 m-B} \geq R\right\}\right) \leq\left\|\chi\left(G-R^{4 m}+1\right)\right\|_{q, r} .
$$

So, we have only to estimate the Sobolev norm on the right hand side.

First we calculate the $L^{q}$-norm.

$$
\left\|\chi\left(G-R^{4 m}+1\right)\right\|_{q} \leq \mathbb{P}\left(\left\{\left\|W^{1}\right\|_{\alpha, 4 m-B} \geq\left(R^{4 m}-1\right)^{1 / 4 m}\right\}\right)^{1 / q} \leq e^{-\left(c_{1} / 2 q\right) R^{2}}
$$

for sufficiently large $R$. It is easy to see that

$$
D\left[\chi\left(G-R^{4 m}+1\right)\right]=\chi^{\prime}\left(G-R^{4 m}+1\right) D G .
$$

Hence,

$$
\begin{aligned}
\left\|D\left[\chi\left(G-R^{4 m}+1\right)\right]\right\|_{q} & \leq\left\|\chi^{\prime}\right\|_{\infty}\left\|I_{\left\{G-R^{4 m}+1 \geq 0\right\}}\right\| D G\left\|_{\mathcal{H}}\right\|_{q} \\
& \leq\left\|\chi^{\prime}\right\|_{\infty}\|D G\|_{2 q} \mathbb{P}\left(\left\{\left\|W^{1}\right\|_{\alpha, 4 m-B} \geq\left(R^{4 m}-1\right)^{1 / 4 m}\right\}\right)^{1 / 2 q} \\
& \leq\left\|\chi^{\prime}\right\|_{\infty}\|D G\|_{2 q} \cdot e^{-\left(c_{1} / 4 q\right) R^{2}}
\end{aligned}
$$

for sufficiently large $R$. From this and Meyer's equivalence, there exists $c_{2}=$ $c_{2}(\alpha, m, q)>0$ such that

$$
\left\|\chi\left(G-R^{4 m}+1\right)\right\|_{1, q} \leq e^{-c_{2} R^{2}}
$$

for sufficiently large $R$. In the same way, we can estimate $D^{k}\left[\chi\left(G-R^{4 m}+1\right)\right]$ for any $k \in \mathbf{R}_{+}$and prove the lemma for $i=1$. 
For $i=2$, just consider

$\tilde{G}(w)=\left\|W^{2}\right\|_{2 \alpha, 2 m-B}^{2 m} \quad$ and $\quad \chi\left(\tilde{G}-R^{4 m}+1\right) \in \mathcal{F}_{q, r}\left(\left\{\left\|W^{2}\right\|_{2 \alpha, 2 m-B}^{1 / 2} \geq R\right\}\right)$.

Then, the same argument works for this case, too.

7.2. Proof of Theorem 2.1 (i): Upper estimate. The aim of this subsection is to prove the upper estimate in Theorem 2.1(i). In this section, we will often use the following fact. For $f, g:(0,1] \rightarrow[0, \infty)$, it holds that $\lim \sup _{\varepsilon \searrow 0} \varepsilon^{2} \log \left(f_{\varepsilon}+g_{\varepsilon}\right) \leq$ $\left[\lim \sup _{\varepsilon \searrow 0} \varepsilon^{2} \log f_{\varepsilon}\right] \vee\left[\lim \sup _{\varepsilon \searrow 0} \varepsilon^{2} \log g_{\varepsilon}\right]$.

Step 1 . We divide the proof into three steps. The first step is to show that

$$
\lim _{R \searrow 0} \limsup _{\varepsilon \searrow 0} \varepsilon^{2} \log \mu_{a, a^{\prime}}^{\varepsilon}\left(B_{R}(X)\right) \leq-I(X), \quad X \in G \Omega_{\alpha, 4 m}^{B}\left(\mathbf{R}^{d}\right),
$$

where

$$
B_{R}(X)=\left\{Y \in G \Omega_{\alpha, 4 m}^{B}\left(\mathbf{R}^{d}\right) \mid\left\|Y^{i}-X^{i}\right\|_{i \alpha, 4 m / i-B}<R^{i} \quad(i=1,2)\right\} .
$$

First, we consider the case $a+\Phi_{0}\left(\tau_{\lambda}(X)\right)_{0,1}^{1} \neq a^{\prime}$. For simplicity we write $\tilde{a}=a+\Phi_{0}\left(\tau_{\lambda}(X)\right)_{0,1}^{1}$. By continuity of Lyons-Itô map, there exist $\varepsilon_{0}>0$ and $R>0$ such that $\left|a+\Phi_{\varepsilon}\left(\tau_{\lambda}(Z)\right)_{0,1}^{1}-\tilde{a}\right| \leq\left|a^{\prime}-\tilde{a}\right| / 3$ holds for all $0 \leq \varepsilon \leq \varepsilon_{0}$ and $Z \in B_{R}(X)$. If we have $\mu_{a, a^{\prime}}^{\varepsilon}\left(B_{R}(X)\right)=0$ for such $\varepsilon$ and $R$, then (7.5) immediately follows for this case.

Let us verify the fact $\mu_{a, a^{\prime}}^{\varepsilon}\left(B_{R}(X)\right)=0$ as follows. Let $\psi: \mathbf{R} \rightarrow[0,1]$ be a smooth even function such that $\psi=1$ on $[0,1]$ and $\psi=0$ on $[2, \infty)$ and non-increasing on $[1,2]$. Set $\psi\left(\left|\left(y_{1}^{\varepsilon}-a^{\prime}\right) / \eta\right|^{2}\right)$, where $\eta:=\left|a^{\prime}-\tilde{a}\right| / 3$. Then, $\delta_{a^{\prime}}\left(y_{1}^{\varepsilon}\right)=\psi\left(\left|\left(y_{1}^{\varepsilon}-a^{\prime}\right) / \eta\right|^{2}\right)$. $\delta_{a^{\prime}}\left(y_{1}^{\varepsilon}\right)$ in $\mathbf{D}_{-\infty}$. By Sugita's theorem, $\theta_{a, a^{\prime}}^{\varepsilon}(d w)=\psi\left(\left|\left(a+\Phi_{\varepsilon}\left(\tau_{\lambda}(\varepsilon W)\right)_{0,1}^{1}-a^{\prime}\right) / \eta\right|^{2}\right)$. $\theta_{a, a^{\prime}}^{\varepsilon}(d w)$, since $a+\Phi_{\varepsilon}\left(\tau_{\lambda}(\varepsilon W)\right)_{0,1}^{1}$ is the $\infty$-quasi-redefinition of $y_{1}^{\varepsilon}=y^{\varepsilon}(1, a, w)$. Hence,

$$
\begin{aligned}
\mu_{a, a^{\prime}}^{\varepsilon}\left(B_{R}(X)\right) & =\theta_{a, a^{\prime}}^{\varepsilon}\left(\left\{w \in \mathcal{W} \mid \varepsilon W \in B_{R}(X)\right\}\right) \\
& =\int_{\mathcal{W}} I_{\left\{\varepsilon W \in B_{R}(X)\right\}} \cdot \psi\left(\left|\left(a+\Phi_{\varepsilon}\left(\tau_{\lambda}(\varepsilon W)\right)_{0,1}^{1}-a^{\prime}\right) / \eta\right|^{2}\right) \theta_{a, a^{\prime}}^{\varepsilon}(d w)=0 .
\end{aligned}
$$

Next we consider the case $a+\Phi_{0}\left(\tau_{\lambda}(X)\right)_{0,1}^{1}=a^{\prime}$. Note that $\left\|\varepsilon W^{1}-X^{1}\right\|_{\alpha, 4 m-B}^{4 m}$ and $\left\|\varepsilon^{2} W^{2}-X^{2}\right\|_{2 \alpha, 2 m-B}^{2 m}$ are in $\hat{\mathcal{C}}_{4 m}$, even if $X \notin S_{2}(\mathcal{H})$. Since their $L^{2}$-norms are bounded in $\varepsilon$, so are their $(q, r)$-Sobolev norms for any $(q, r)$. Note also that $\left\|D^{r} y_{1}^{\varepsilon}\right\|_{q}$ is bounded in $\varepsilon$ for any $(q, r)$. Set $G\left(u_{1}, \ldots, u_{n}\right)=\prod_{j=1}^{n}\left(u_{j}-a_{j}^{\prime}\right)^{+}$. This is a continuous function from $\mathbf{R}^{n}$ to $\mathbf{R}$ with polynomial growth and satisfies $\partial_{1}^{2} \cdots \partial_{n}^{2} G(u)=\delta_{a^{\prime}}(u)$ in the sense of Schwartz distributions.

Then, we have

$$
\begin{aligned}
\mu_{a, a^{\prime}}^{\varepsilon}\left(B_{R}(X)\right) & =\theta_{a, a^{\prime}}^{\varepsilon}\left(\left\{w \in \mathcal{W} \mid \varepsilon W \in B_{R}(X)\right\}\right) \\
& \leq \mathbb{E}\left[\prod_{i=1}^{2} \psi\left(\left\|\varepsilon^{i} W^{i}-X^{i}\right\|_{i \alpha, 4 m / i-B}^{4 m / i} / R^{4 m}\right) \cdot\left(\partial_{1}^{2} \cdots \partial_{n}^{2} G\right)\left(y_{1}^{\varepsilon}\right)\right] .
\end{aligned}
$$

Now we use integration by parts as in (4.2)-(4.3). Then, the right hand side of (17.6) is equal to a finite sum of the following form:

$$
\begin{aligned}
\sum_{j, k} \mathbb{E}\left[F_{j, k}^{\varepsilon}\right. & \cdot \psi^{(j)}\left(\left\|\varepsilon W^{1}-X^{1}\right\|_{\alpha, 4 m-B}^{4 m} / R^{4 m}\right) \\
& \left.\times \psi^{(k)}\left(\left\|\varepsilon^{2} W^{2}-X^{2}\right\|_{2 \alpha, 2 m-B}^{2 m} / R^{4 m}\right) G\left(y_{1}^{\varepsilon}\right)\right] .
\end{aligned}
$$


Here, $F_{j, k}^{\varepsilon}(w)=F_{j, k}(\varepsilon, w)$ is a polynomial in components of (i) $y_{1}^{\varepsilon}$ and its derivatives, (ii) $\left\|\varepsilon^{i} W^{i}-X^{i}\right\|_{i \alpha, 4 m / i-B}^{4 m / i}$ and its derivatives, (iii) $\tau(\varepsilon)$, which is a Malliavin covariance matrix of $y_{1}^{\varepsilon}$, and (iv) $\gamma(\varepsilon)=\tau(\varepsilon)^{-1}$. Note that derivatives of $\gamma(\varepsilon)$ do not appear.

By the uniform non-degeneracy of the Malliavin covariance matrix of $\left(y_{1}^{\varepsilon}-a\right) / \varepsilon$, there exists $l>0$ such that $\gamma(\varepsilon)$ is $O\left(\varepsilon^{-l}\right)$ in any $L^{q}$. In turn, this implies that there exists $l>0$ such that $F_{j, k}^{\varepsilon}$ is $O\left(\varepsilon^{-l}\right)$ in any $L^{q}$. (Here and in what follows, $l$ may change from line to line.)

Take $q, q^{\prime} \in(1, \infty)$ so that $1 / q+1 / q^{\prime}=1$. By Hölder's inequality, the right hand side of (7.7) is dominated by

$$
\begin{gathered}
C \varepsilon^{-l} \mu\left(\left\|\varepsilon^{i} W^{i}-X^{i}\right\|_{i \alpha, 4 m / i-B}^{1 / i} \leq 2^{\frac{1}{m}} R(i=1,2)\right)^{\frac{1}{q^{\prime}}} \\
=C \varepsilon^{-l} \mu\left(\varepsilon W \in B_{2 \frac{1}{m}}(X)\right)^{\frac{1}{q^{\prime}}} .
\end{gathered}
$$

Here, $C=C_{q, q^{\prime}}>0$ is a constant independent of $\varepsilon$. By the large deviation principle of Schilder-type for the law of $\varepsilon W$ on $G \Omega_{\alpha, 4 m}^{B}\left(\mathbf{R}^{d}\right)$,

$$
\limsup _{\varepsilon \searrow 0} \varepsilon^{2} \log \mu_{a, a^{\prime}}^{\varepsilon}\left(B_{R}(X)\right) \leq-\frac{1}{q^{\prime}} \inf \left\{\|h\|_{\mathcal{H}}^{2} / 2 \mid h \in \mathcal{H}, S_{2}(h) \in B_{2^{1 / m} R}(X)\right\} .
$$

Letting $q^{\prime} \searrow 1$, we have

$$
\begin{aligned}
\limsup _{\varepsilon \searrow 0} \varepsilon^{2} \log \mu_{a, a^{\prime}}^{\varepsilon}\left(B_{R}(X)\right) & \leq-\inf \left\{\|h\|_{\mathcal{H}}^{2} / 2 \mid h \in \mathcal{H}, S_{2}(h) \in B_{2^{1 / m} R}(X)\right\} \\
& =-\inf \left\{I_{S c h}(Y) \mid Y \in B_{2^{1 / m} R}(X)\right\} .
\end{aligned}
$$

Since the rate function $I_{S c h}: G \Omega_{\alpha, 4 m}^{B}\left(\mathbf{R}^{d}\right) \rightarrow[0, \infty]$ is lower semicontinuous, the limit of the right hand side as $R \searrow 0$ is dominated by $-I_{S c h}(X)$. This proves (7.5). (Here, $I_{S c h}\left(S_{2}(h)\right)=\|h\|_{\mathcal{H}}^{2} / 2$ and $I_{S c h}(X)=\infty$ if $X$ is not lying above any $h \in \mathcal{H}$.)

Step 2. The second step is to prove the upper bound in Theorem 2.1 (i) when $A$ is a compact set. Let $N \in \mathbf{N}_{+}$. For any $Y \in A$, take $R=R_{N, Y}>0$ small enough so that

$$
\limsup _{\varepsilon \searrow 0} \varepsilon^{2} \log \mu_{a, a^{\prime}}^{\varepsilon}\left(B_{R}(Y)\right) \leq \begin{cases}-N & \text { (if } I(Y)=\infty), \\ -I(Y)+N^{-1} & \text { (if } I(Y)<\infty) .\end{cases}
$$

The union of such open balls over $Y \in A$ covers the compact set $A$. Hence, there are finitely many $Y_{1}, \ldots, Y_{k}$ such that $A \subset \bigcup_{i=1}^{k} B_{R_{i}}\left(Y_{i}\right)$, where $R_{i}=R\left(N, Y_{i}\right)$. By using the remark in the beginning of this section, we see that

$$
\begin{aligned}
\limsup _{\varepsilon \searrow 0} \varepsilon^{2} \log \mu_{a, a^{\prime}}^{\varepsilon}(A) & \leq(-N) \vee \max \left\{-I\left(Y_{i}\right)+N^{-1} \mid 1 \leq i \leq k, I\left(Y_{i}\right)<\infty\right\} \\
& \leq(-N) \vee\left(-\inf _{h \in A \cap \mathcal{K}^{a, a^{\prime}}}\|h\|_{\mathcal{H}}^{2} / 2+N^{-1}\right) .
\end{aligned}
$$

Letting $N \rightarrow \infty$, we obtain

$$
\limsup _{\varepsilon \searrow 0} \varepsilon^{2} \log \mu_{a, a^{\prime}}^{\varepsilon}(A) \leq-\inf \left\{\|h\|_{\mathcal{H}}^{2} / 2 \mid h \in \mathcal{K}^{a, a^{\prime}}, S_{2}(h) \in A\right\} .
$$

Thus, we have obtained the upper estimate for the compact case. 
Step 3. In the final step, we verify the case when $A$ is a closed set. Take $\alpha^{\prime} \in$ $(\alpha, 1 / 2)$ so that $\left(\alpha^{\prime}, m\right)$ still satisfies (2.3). As in the proof of Proposition 7.5 , let $B_{R}^{\prime}(H)=T_{h}\left(B_{R}\right)$ be the "ball" of radius $R>0$ centered at $H$ in $\left(\alpha^{\prime}, 4 m\right)$-Besov geometric rough path space. (We will use the same symbol by abusing notation.) Note that this is precompact in $G \Omega_{\alpha, 4 m}^{B}\left(\mathbf{R}^{d}\right)$ by Proposition 3.2 ,

Choose any $\bar{h} \in \mathcal{K}^{a, a^{\prime}} \cap \mathcal{W}^{*}$. For sufficiently large $R>0$,

$$
\begin{aligned}
\mu_{a, a^{\prime}}^{\varepsilon}\left(B_{R}^{\prime}(\bar{H})^{c}\right)= & \int_{\mathcal{W}} I_{T_{\bar{h}}\left(B_{R}\right)^{c}}(\varepsilon W) \theta_{a, a^{\prime}}^{\varepsilon}(d w) \\
= & \varepsilon^{-n} \int_{\mathcal{W}} \exp \left(-\frac{\langle\bar{h}, w\rangle}{\varepsilon}-\frac{\|\bar{h}\|_{\mathcal{H}}^{2}}{2 \varepsilon^{2}}\right) I_{\left\{\left\|W^{i}\right\|_{i \alpha^{\prime}, 4 m / i-B}^{1 / i}<R / \varepsilon(i=1,2)\right\}^{c}} \nu^{\varepsilon}(d w) \\
\leq & \varepsilon^{-n} e^{\|\bar{h}\|_{\mathcal{H}}^{2} / 2 \varepsilon^{2}} \mathbb{E}\left[\exp \left(-\frac{\langle 2 \bar{h}, w\rangle}{\varepsilon}-\frac{\|2 \bar{h}\|_{\mathcal{H}}^{2}}{2 \varepsilon^{2}}\right) \delta_{0}\left(\frac{y_{1}^{\varepsilon, h}-a^{\prime}}{\varepsilon}\right)\right]^{1 / 2} \\
& \times\left\{\sum_{i=1,2} \nu^{\varepsilon}\left(\left\{\left\|W^{i}\right\|_{i \alpha^{\prime}, 4 m / i-B}^{1 / i} \geq R / \varepsilon\right)\right\}^{1 / 2} .\right.
\end{aligned}
$$

As we proved in (the proof of) Proposition 7.5 and Lemma 7.6, the last factor above is dominated by $2 e^{-c_{1} R^{2} / \varepsilon^{2}}$ for some constant $c_{1}>0$. Since, for some $q^{\prime}, r,\left\|\delta_{0}\left(\left(y_{1}^{\varepsilon, \bar{h}}-a^{\prime}\right) / \varepsilon\right)\right\|_{q^{\prime},-r}$ is bounded in $\varepsilon$, it suffices to estimate the $(q, r)$-Sobolev norm of the Cameron-Martin density function $\exp \left(-\langle 2 \bar{h}, w\rangle / \varepsilon-\|2 \bar{h}\|_{\mathcal{H}}^{2} / 2 \varepsilon^{2}\right)$. Using Hölder's inequality, we can easily see that its $L^{q}$-norm is dominated by $e^{2(q-1)\|\bar{h}\|_{\mathcal{H}}^{2} / \varepsilon^{2}}$. We can also estimate $L^{q}$-norms of its derivatives and, by using Meyer's equivalence, we have

$$
\left\|\exp \left(\frac{\langle 2 \bar{h}, w\rangle}{\varepsilon}-\frac{\|2 \bar{h}\|_{\mathcal{H}}^{2}}{2 \varepsilon^{2}}\right)\right\|_{q, r} \leq c_{2} \varepsilon^{-c_{3}}\left(1+\|h\|_{\mathcal{H}}\right)^{c_{4}} e^{2(q-1)\|\bar{h}\|_{\mathcal{H}}^{2} / \varepsilon^{2}}
$$

for some positive constants $c_{j}=c_{j, q, r}(j=2,3,4)$, which are independent of $\varepsilon$. Therefore, we have

$$
\limsup _{\varepsilon \searrow 0} \varepsilon^{2} \log \mu_{a, a^{\prime}}^{\varepsilon}\left(B_{R}^{\prime}(\bar{H})^{c}\right) \leq-c_{1} R^{2}+\left(q-\frac{1}{2}\right)\|\bar{h}\|_{\mathcal{H}}^{2} .
$$

Note that the right hand side can be made arbitrarily small if we take $R$ large enough.

Now let $A$ be a closed set such that $A \cap S_{2}\left(\mathcal{K}^{a, a^{\prime}}\right) \neq \emptyset$. Take $R>0$ so that

$$
-c_{1} R^{2}+\left(q-\frac{1}{2}\right)\|\bar{h}\|_{\mathcal{H}}^{2}<-\inf _{h \in A \cap \mathcal{K}^{a, a^{\prime}}}\|h\|_{\mathcal{H}}^{2} / 2 .
$$

Decompose $A=\overline{B_{R}^{\prime}(\bar{H})} \cap A+\left(\overline{B_{R}^{\prime}(\bar{H})}\right)^{c} \cap A$ into a union of two disjoint sets. The second set is included in $B_{R}^{\prime}(\bar{H})^{c}$. The first set in the disjoint union is compact and, due to the previous step, we have

$$
\begin{aligned}
\limsup _{\varepsilon \searrow 0} \varepsilon^{2} \log \mu_{a, a^{\prime}}^{\varepsilon}\left(\overline{B_{R}^{\prime}(\bar{H})} \cap A\right) & \leq-\inf \left\{\|h\|_{\mathcal{H}}^{2} / 2 \mid h \in \mathcal{K}^{a, a^{\prime}}, S_{2}(h) \in A \cap \overline{B_{R}^{\prime}(\bar{H})}\right\} \\
& \leq-\inf \left\{\|h\|_{\mathcal{H}}^{2} / 2 \mid h \in \mathcal{K}^{a, a^{\prime}}, S_{2}(h) \in A\right\} .
\end{aligned}
$$

By the remark in the beginning of this section, we obtain the upper estimate in this case. The case $A \cap S_{2}\left(\mathcal{K}^{a, a^{\prime}}\right)=\emptyset$ can be done in a similar way and is actually easier. This completes the proof of Theorem 2.1 (i). 


\section{REFERENCES}

[1] Shigeki Aida, Vanishing of one-dimensional $L^{2}$-cohomologies of loop groups, J. Funct. Anal. 261 (2011), no. 8, 2164-2213, DOI 10.1016/j.jfa.2011.06.003. MR2824575

[2] I. Bailleul, Large deviation principle for bridges of degenerate diffusion processes. Preprint 2013. Arxiv Math: 1303.2854.

[3] P. Baldi and M. Sanz, Une remarque sur la théorie des grandes déviations (French), Séminaire de Probabilités, XXV, Lecture Notes in Math., vol. 1485, Springer, Berlin, 1991, pp. 345-348, DOI 10.1007/BFb0100868. MR.1187792 (93k:60065)

[4] Amir Dembo and Ofer Zeitouni, Large deviations techniques and applications, 2nd ed., Applications of Mathematics (New York), vol. 38, Springer-Verlag, New York, 1998. MR 1619036 (99d:60030)

[5] S. Dereich, Rough paths analysis of general Banach space-valued Wiener processes, J. Funct. Anal. 258 (2010), no. 9, 2910-2936, DOI 10.1016/j.jfa.2010.01.018. MR2595729 (2011e:60192)

[6] Avner Friedman, Stochastic differential equations and applications. Vol. 2, Academic Press [Harcourt Brace Jovanovich Publishers], New York, 1976. Probability and Mathematical Statistics, Vol. 28. MR0494491 (58 \#13350b)

[7] Peter Friz and Harald Oberhauser, A generalized Fernique theorem and applications, Proc. Amer. Math. Soc. 138 (2010), no. 10, 3679-3688, DOI 10.1090/S0002-9939-2010-10528-2. MR2661566 (2011i:60065)

[8] Peter Friz and Nicolas Victoir, Approximations of the Brownian rough path with applications to stochastic analysis (English, with English and French summaries), Ann. Inst. H. Poincaré Probab. Statist. 41 (2005), no. 4, 703-724, DOI 10.1016/j.anihpb.2004.05.003. MR2144230 (2007e:60018)

[9] P. Friz and N. Victoir, Large deviation principle for enhanced Gaussian processes. Ann. Inst. H. Poincaré Probab. Statist. 43 (2007), no. 6, 775-785.

[10] Peter K. Friz and Nicolas B. Victoir, Multidimensional stochastic processes as rough paths, Cambridge Studies in Advanced Mathematics, vol. 120, Cambridge University Press, Cambridge, 2010. Theory and applications. MR2604669 (2012e:60001)

[11] Fuqing Gao and Jiagang Ren, Large deviations for stochastic flows and their applications, Sci. China Ser. A 44 (2001), no. 8, 1016-1033, DOI 10.1007/BF02878977. MR.1857556 (2002j:60099)

[12] Y. Higuchi, Master Theses (in Japanese), Graduate School of Engineering Sciences, Osaka University, 2006.

[13] Pei Hsu, Brownian bridges on Riemannian manifolds, Probab. Theory Related Fields 84 (1990), no. 1, 103-118, DOI 10.1007/BF01288561. MR1027823 (90m:58227)

[14] Nobuyuki Ikeda and Shinzo Watanabe, Stochastic differential equations and diffusion processes, 2nd ed., North-Holland Mathematical Library, vol. 24, North-Holland Publishing Co., Amsterdam, 1989. MR1011252 (90m:60069)

[15] Yuzuru Inahama, Quasi-sure existence of Brownian rough paths and a construction of Brownian pants, Infin. Dimens. Anal. Quantum Probab. Relat. Top. 9 (2006), no. 4, 513-528, DOI 10.1142/S0219025706002512. MR2282717 (2008d:60069)

[16] Yuzuru Inahama and Hiroshi Kawabi, Large deviations for heat kernel measures on loop spaces via rough paths, J. London Math. Soc. (2) 73 (2006), no. 3, 797-816, DOI 10.1112/S0024610706022654. MR2241981 (2007i:58047)

[17] Shigeo Kusuoka and Daniel W. Stroock, Precise asymptotics of certain Wiener functionals, J. Funct. Anal. 99 (1991), no. 1, 1-74, DOI 10.1016/0022-1236(91)90051-6. MR.1120913 (93a:60085)

[18] M. Ledoux, Z. Qian, and T. Zhang, Large deviations and support theorem for diffusion processes via rough paths, Stochastic Process. Appl. 102 (2002), no. 2, 265-283, DOI 10.1016/S0304-4149(02)00176-X. MR.1935127 (2003m:60152)

[19] Terry J. Lyons, Differential equations driven by rough signals, Rev. Mat. Iberoamericana 14 (1998), no. 2, 215-310, DOI 10.4171/RMI/240. MR1654527(2000c:60089)

[20] Terry Lyons and Zhongmin Qian, System control and rough paths, Oxford Mathematical Monographs, Oxford University Press, Oxford, 2002. Oxford Science Publications. MR2036784 (2005f:93001) 
[21] Paul Malliavin, Stochastic analysis, Grundlehren der Mathematischen Wissenschaften [Fundamental Principles of Mathematical Sciences], vol. 313, Springer-Verlag, Berlin, 1997. MR:1450093 (99b:60073)

[22] Paul Malliavin and David Nualart, Quasi-sure analysis of stochastic flows and Banach space valued smooth functionals on the Wiener space, J. Funct. Anal. 112 (1993), no. 2, 287-317, DOI 10.1006/jfan.1993.1034. MR1213140 (94g:60102)

[23] Annie Millet and Marta Sanz-Solé, Large deviations for rough paths of the fractional Brownian motion (English, with English and French summaries), Ann. Inst. H. Poincaré Probab. Statist. 42 (2006), no. 2, 245-271, DOI 10.1016/j.anihpb.2005.04.003. MR2199801 (2007e:60027)

[24] Jia Gang Ren, Analyse quasi-sûre des équations différentielles stochastiques (French, with English summary), Bull. Sci. Math. 114 (1990), no. 2, 187-213. MR1056161 (91m:60102)

[25] Ichiro Shigekawa, Stochastic analysis, Translations of Mathematical Monographs, vol. 224, American Mathematical Society, Providence, RI, 2004. Translated from the 1998 Japanese original by the author; Iwanami Series in Modern Mathematics. MR2060917 (2005k:60002)

[26] Hiroshi Sugita, Positive generalized Wiener functions and potential theory over abstract Wiener spaces, Osaka J. Math. 25 (1988), no. 3, 665-696. MR969026 (90c:60036)

[27] Hiroshi Sugita, Hu-Meyer's multiple Stratonovich integral and essential continuity of multiple Wiener integral, Bull. Sci. Math. 113 (1989), no. 4, 463-474. MR 1029620

[28] S. Takanobu and S. Watanabe, Asymptotic expansion formulas of the Schilder type for a class of conditional Wiener functional integrations, asymptotics (Sanda/Kyoto, 1990), Pitman Res. Notes Math. Ser., vol. 284, Longman Sci. Tech., Harlow, 1993, pp. 194-241. MR.1354169 (96m:60128)

[29] Shinzo Watanabe, Analysis of Wiener functionals (Malliavin calculus) and its applications to heat kernels, Ann. Probab. 15 (1987), no. 1, 1-39. MR877589 (88h:60111)

[30] Shinzo Watanabe, Itô calculus and Malliavin calculus, Stochastic analysis and applications, Abel Symp., vol. 2, Springer, Berlin, 2007, pp. 623-639, DOI 10.1007/978-3-540-70847-6_29. MR2397809 (2009f:60069)

Graduate School of Mathematics, Nagoya University, Furocho, Chikusa-Ku, Nagoya 464-8602, JAPAN

E-mail address: inahama@math.nagoya-u.ac.jp 